\title{
Massive deformations of Type IIA theory within double field theory
}

\author{
Aybike Çatal-Özer \\ Department of Mathematics, Istanbul Technical University, \\ Maslak 34469, Istanbul, Turkey \\ E-mail: ozerayb@itu.edu.tr
}

ABSTRACT: We obtain massive deformations of Type IIA supergravity theory through duality twisted reductions of Double Field Theory (DFT) of massless Type II strings. The mass deformation is induced through the reduction of the DFT of the RR sector. Such reductions are determined by a twist element belonging to $\operatorname{Spin}^{+}(10,10)$, which is the duality group of the DFT of the RR sector. We determine the form of the twists and give particular examples of twists matrices, for which a massive deformation of Type IIA theory can be obtained. In one of the cases, requirement of gauge invariance of the RR sector implies that the dilaton field must pick up a linear dependence on one of the dual coordinates. In another case, the choice of the twist matrix violates the weak and the strong constraints explicitly in the internal doubled space.

KeYworDs: Flux compactifications, String Duality

ARXiv EPrint: 1706.08883 


\section{Contents}

1 Introduction 1

2 Review of duality twisted reductions of DFT 3

3 Double field theory of massive Type IIA theory 9

$\begin{array}{lll}3.1 \text { Fluxes } & 11\end{array}$

3.2 Determining the twist element that gives rise to massive IIA 13

$\begin{array}{lll}3.2 .1 & \text { Twists with } S(y, \tilde{y})=S_{A}(\tilde{y}), \beta=0 & 14\end{array}$

$\begin{array}{ll}3.2 .2 & \text { Twists with } S(y, \tilde{y})=S_{\beta}(\tilde{y}), A=0 \\ 3.2 .3 & 15\end{array}$

$\begin{array}{lll}3.2 .3 & \text { Mixed twists } S(y, \tilde{y})=S_{\beta}(y) . S_{A}(\tilde{y}) & 16\end{array}$

$\begin{array}{lll}3.3 & \text { The reduced action } & 18\end{array}$

$\begin{array}{llr}4 & \text { Conclusion and outlook } & 19\end{array}$

A The NS-NS sector $\quad 21$

A.1 Twists with non-vanishing $f_{1}^{12} \quad 22$

A.2 Twists with non-vanishing $f^{123} \quad 23$

$\begin{array}{ll}\text { B The mixed twist matrix } & 23\end{array}$

\section{Introduction}

Double Field Theory (DFT) is a field theory defined on a doubled space, which implements the $\mathrm{O}(d, d)$ T-duality symmetry of string theory as a manifest symmetry. In addition to the standard space-time coordinates, the doubled space also includes dual coordinates, which are associated with the winding excitations of closed string theory on backgrounds with non-trivial cycles. The DFT action was constructed in [1-4], building on earlier work [5-13]. The consistency of the action requires the imposition of a set of constraints, called the weak and the strong constraints. The strong constraint implies that locally all fields and parameters of the theory depend only on half of the coordinates. In a certain frame called the supergravity frame, in which none of the fields depend on the dual coordinates associated with the winding excitations, the DFT action constructed by [1-4] reduces to the action of the NS-NS sector of massless string theory. The weak and the strong constraints are satisfied in a trivial way for this choice of frame. The gauge transformation rules of DFT are governed by the so-called C-bracket, which is an $\mathrm{O}(d, d)$ covariantization of the Courant bracket in generalized geometry of Hitchin [14-16]. In later work [17, 18], the DFT action of the RR sector of Type II string theory was also constructed, ${ }^{1}$ where it was shown

\footnotetext{
${ }^{1}$ An alternative formulation of the RR sector, called the semi-covariant formulation is given in the papers $[19,20]$.
} 
that in the supergravity frame this new action reduces to the democratic formulation of the RR sector of Type II supergravity. The fields in the RR sector are chiral spinor fields, which form a representation of Pin $(10,10)$. The action does not possess the full Pin $(10,10)$ symmetry. The chirality condition and the existence of a self-duality condition which must supplement the action reduces the symmetry group to $\operatorname{Spin}^{+}(10,10)$.

In the interesting paper [21], the action constructed by [17] was shown to give rise to massive Type IIA theory of [22] in a certain frame, in which the RR one-form of Type IIA is allowed to pick up a linear dependence on one of the dual coordinates. Unlike the supergravity frame, in which the action reduces to the democratic formulation of massless type II theories, the strong constraint is violated in the frame considered in [21]. However, the weak constraint is still respected. To be more precise, the anzats (through which the RR fields pick up their coordinate dependencies) of [21] is not in the most general possible form allowed by the weak constraint, and owes its consistency to the fact that the dependence on the dual coordinates is linear. Consistency of the theory also requires a reformulation of the gauge transformations on the RR fields, in such a way that the linear dependence on the dual coordinates would drop out. The work of [21] is particularly interesting, as it also addresses the challenge of relaxing the strong constraint.

There has been other attempts to relax the strong and the weak constraints by various groups. One line of work in this direction involves formulating the DFT action on group manifolds rather than toroidal backgrounds, as initiated by $[32,33]$. Possibility of relaxing the strong constraint, albeit partially, also comes from the study of duality twisted reductions of DFT. As mentioned above, DFT comes equipped with the large duality group $\mathrm{O}(d, d)$, which makes it possible to introduce a duality twisted reduction anzats (also called generalized Scherk-Schwarz type anzats [23, 24]) for the fields and gauge parameters of the theory. This line of work has been pursued by many groups so far [25-31]. An interesting aspect of these works is that one never needs to impose the weak and the strong constraints on the doubled internal space. This feature was made explicit in [27], where they gave the set of conditions to be satisfied for the consistency of the twisted reduction and showed that these constituted a weaker set of constraints compared with the constraints of DFT.

The linear anzats of [21] and the Scherk-Schwarz type anzatse mentioned above are two prominent examples with success towards the aim of relaxing the constraints of DFT. This has motivated us to study these two approaches together. In this paper, our aim is to explore whether massive deformations of Type IIA theory can be obtained through duality twisted reductions of DFT. Indeed, we show that a mass deformation can be induced through the reduction of the DFT of the RR sector. Such reductions have been studied recently [34] and are determined by a twist element belonging to $\operatorname{Spin}^{+}(10,10)$, which is the duality group of the DFT of the RR sector. We determine the form of the twists and give particular examples of twists matrices, for which a massive deformation of Type IIA theory can be obtained. In one of the cases, requirement of gauge invariance of the RR sector implies that the dilaton field must pick up a linear dependence on one of the dual coordinates. We find this aspect particularly interesting, given that reductions with nontrivial dilaton anzats have received some interest recently, in attempts to understand the recently discovered generalized supergravity equations [37] within DFT [38-40]. In another 
case, the choice of the twist matrix violates the weak and the strong constraints explicitly in the internal doubled space.

The plan of the paper is as follows. In the next section, we give a brief review of the Double Field Theory of Type II strings and its reduction with duality twists. Section 3 is the main section of the paper, where we determine the twist element, which gives rise to the massive deformation of Type IIA theory. In order to explain how the mass deformation arises, we start by a more detailed explanation of the DFT action of the RR sector, focusing on its formulation in terms of Mukai pairing, which is a $\operatorname{Spin}(d, d)$ invariant bilinear form on the space of spinors [41]. Then, we continue with the computation of fluxes that determine the deformations of the RR sector. This makes it possible to determine the possible forms of twists that could give rise to a mass deformation in the subsequent subsections. We end the paper with discussions and outlook in section 4 . In the main body of the paper, we mainly focus on the RR sector, as the mass deformation is induced through the reduction of this sector. The appendix A collects useful information about the NS-NS sector and its deformation through duality twisted reduction anzatse.

\section{Review of duality twisted reductions of DFT}

In this section, we give a very brief review of DFT and its duality twisted reduction. Our aim here is to set the notation and present the formulas needed in the main section 3 . For a more complete introduction to these topics, see [3, 17, 26-28, 34].

In DFT, the number of space-time coordinates is doubled by introducing dual coordinates $\tilde{x}_{i}$, which are associated with winding excitations of string theory on backgrounds with non-trivial cycles. The standard and the dual coordinates combine to form an $\mathrm{O}(d, d)$ vector transforming as:

$$
X^{\prime M}=h^{M}{ }_{N} X^{N}, \quad X^{M}=\left(\begin{array}{c}
\tilde{x}_{i} \\
x^{i}
\end{array}\right)
$$

Here $h^{M}{ }_{N}$ is a general $\mathrm{O}(d, d)$ matrix. We raise and lower indices by the $\mathrm{O}(d, d)$ invariant metric $\eta$, and hence $X_{M}=\eta_{M N} X^{N}$. The $\mathrm{O}(d, d)$ invariant constraints are

$$
\partial^{M} \partial_{M} A=\eta^{M N} \partial_{M} \partial_{N} A=0, \quad \partial^{M} A \partial_{M} B=0, \quad \eta^{M N}=\left(\begin{array}{ll}
0 & 1 \\
1 & 0
\end{array}\right),
$$

where $A$ and $B$ represent any fields or parameters of the theory. The first of the above constraints is called the weak constraint. It follows from the level matching constraint in closed string theory. The second constraint is stronger and is called the strong constraint. It is too strong actually, as it implies that all fields and gauge parameters of the theory can depend only on half of the coordinates.

The DFT action of Type II strings is $[4,17,18]$

$$
\mathcal{S}=\int d x d \tilde{x}\left(\mathcal{L}_{\mathrm{NS}-\mathrm{NS}}+\mathcal{L}_{\mathrm{RR}}\right)
$$

where

$$
\mathcal{L}_{\mathrm{NS}-\mathrm{NS}}=e^{-2 d} \mathcal{R}(\mathcal{H}, d) \quad \text { and } \quad \mathcal{L}_{\mathrm{RR}}=\frac{1}{4}(\not \partial \chi)^{\dagger} \mathbb{S} \not \partial \chi=\frac{1}{4}\left\langle\not \partial \chi, C^{-1} \mathbb{S} \not \partial \chi\right\rangle .
$$


Here, \langle\rangle is the Mukai pairing, which is a $\operatorname{Spin}(d, d)$ invariant bilinear form on the space of spinors $[16,41]$. The action (2.3) has to be supplemented by the following self-duality constraint

$$
\not \partial \chi=-\mathcal{K} \not \partial \chi, \quad \mathcal{K} \equiv C^{-1} \mathbb{S} .
$$

In the supergravity frame in which $\tilde{\partial}^{i}=0$, the first term in the above action reduces to the standard NS-NS action for the massless fields of string theory and the second term reduces to the RR sector of the democratic formulation of Type II supergravity theories. For this reason, the first term is called the DFT action of the NS-NS sector of string theory, whereas the second term is referred to as the DFT action of the RR sector.

The $\mathcal{R}(\mathcal{H}, d)$ in $(2.4)$ is the generalized Ricci scalar [4]. Its explicit form can be found in (A.1). The generalized Ricci scalar is defined in terms of the generalized metric $\mathcal{H}$ and the generalized dilaton field $d$. We present these generalized $\mathrm{O}(d, d)$ tensors below:

$$
\mathcal{H}_{M N}=\left(\begin{array}{cc}
\mathcal{H}^{i j} & \mathcal{H}^{i}{ }_{j} \\
\mathcal{H}_{i}{ }^{j} & \mathcal{H}_{i j}
\end{array}\right)=\left(\begin{array}{cc}
g^{i j} & -g^{i k} b_{k j} \\
b_{i k} g^{k j} & g_{i j}-b_{i k} g^{k l} b_{l j}
\end{array}\right), \quad e^{-2 d}=\sqrt{g} e^{-2 \phi},
$$

where, $g=|\operatorname{det} g|$. The generalized metric $\mathcal{H}$ transforms covariantly under $\mathrm{O}(d, d)$ transformations and the generalized dilaton field is invariant under such transformations.

The field $\mathbb{S}$ in the Ramond-Ramond sector is the spinor representative of the generalized metric $\mathcal{H}$ under the double covering homomorphism $\rho: \operatorname{Spin}(d, d) \rightarrow \mathrm{SO}(d, d)$, that is, $\rho(\mathbb{S})=\mathcal{H}$. Since the generalized metric $\mathcal{H}$ is in the coset $\mathrm{SO}^{-}(d, d)$ in Lorentzian signature, the spinor field $\mathbb{S}$ it lifts to, is an element of $\operatorname{Spin}^{-}(d, d)$. The field $\chi$, which is the other dynamical field in the DFT of the RR sector is a chiral spinor field. It encodes all the (modified) p-form fields in the RR sector. The chirality of $\chi$ determines whether the corresponding theory is Type IIA or Type IIB string theory. The former corresponds to negative chirality of $\chi$, whereas the latter involves the spinor field $\chi$ with postive chirality. The operator $\not \partial$ in the action (2.3) is the generalized Dirac operator. It is defined as

$$
\not \partial \equiv \frac{1}{\sqrt{2}} \Gamma^{M} \partial_{M}=\frac{1}{\sqrt{2}}\left(\Gamma^{i} \partial_{i}+\Gamma_{i} \tilde{\partial}^{i}\right) \equiv \psi^{i} \partial_{i}+\psi_{i} \tilde{\partial}^{i} .
$$

Here the Gamma matrices $\Gamma^{M}=\left(\Gamma_{i}, \Gamma^{i}\right)$ are the matrix representations of the generators of the Clifford algebra $C L\left(R^{2 d}, \eta\right)$ :

$$
\left\{\Gamma^{M}, \Gamma^{N}\right\}=2 \eta^{M N} .
$$

The labelling is such that the first $d$ elements span a maximally isotropic subsace with respect to the metric $\eta$ and the remaining elements span the orthogonal complement. Then one has

$$
\left\{\Gamma_{i}, \Gamma^{j}\right\}=2 \delta_{i}^{j}, \quad\left\{\Gamma_{i}, \Gamma_{j}\right\}=0, \quad\left\{\Gamma^{i}, \Gamma^{j}\right\}=0,
$$

The algebra elements $\Gamma^{M}$ do not lie in the associated group $\operatorname{Spin}(d, d)$, so it is more appropriate to work with $\psi^{M}=\frac{1}{\sqrt{2}} \Gamma^{M}$, which do lie in the spin group. ${ }^{2}$ The spinorial action of the Clifford algebra elements $\Gamma^{M}$ on spinor fields $\chi$ is best understood when one identifies

\footnotetext{
${ }^{2} \psi^{M}$ satisfy $\psi^{M}\left(\psi^{M}\right)^{*}= \pm 1$, so they lie in the spinor group [16, 34].
} 
the spinor fields with (not necessarily homogenous) differential forms (or with polyforms, as is commonly called in physics literature) of the exterior algebra $\bigwedge^{\bullet} R^{d}[16,17,34] .^{3}$ Then, if one writes the exterior algebra element $\chi$ as

$$
\chi(x, \tilde{x})=\sum_{p} \frac{1}{p !} C_{i_{1} \ldots i_{p}}(x, \tilde{x}) \psi^{i_{1}} \ldots \psi^{i_{p}},
$$

then $\psi^{i}$ acts on $\chi$ by wedge product, whereas $\psi_{i}$ acts by contraction. More, precisely, one has

$$
\psi^{i} \cdot \chi=\psi^{i} \wedge \chi, \quad \psi_{i} \cdot \chi=i_{\psi_{i}} \chi
$$

where one defines $i_{\psi_{i}} \psi^{j}=\delta_{i}{ }^{j}$. For more details see [17, 34].

The gauge transformation rules of the DFT action are determined by the generalized Lie derivative $\hat{\mathcal{L}}$. Let us define the $\mathrm{O}(d, d)$ vector $\xi^{M}=\left(\tilde{\xi}_{i}, \xi^{i}\right)$ as the parameter of the gauge transformations. Then, we have [2]

$$
\begin{aligned}
\delta_{\xi} \chi & =\widehat{\mathcal{L}}_{\xi} \chi \equiv \xi^{M} \partial_{M} \chi+\frac{1}{\sqrt{2}} \not \xi^{M} \Gamma_{M} \chi \\
\delta_{\xi} \mathcal{K} & =\xi^{M} \partial_{M} \mathcal{K}+\frac{1}{2}\left[\Gamma^{P Q}, \mathcal{K}\right] \partial_{P} \xi_{Q},
\end{aligned}
$$

in the RR sector, where $\Gamma^{P Q} \equiv \frac{1}{2}\left[\Gamma^{P}, \Gamma^{Q}\right]$. The double field theory version of the abelian gauge symmetry of p-form gauge fields is

$$
\delta_{\lambda} \chi=\not \partial \lambda=\frac{1}{\sqrt{2}} \Gamma^{M} \partial_{M} \lambda .
$$

Here, $\lambda$ is a space-time dependent spinor. The gauge transformation rules for the NS-NS sector is given in appendix A. The gauge algebra closes with respect to the C-bracket:

$$
\left[\xi_{1}, \xi_{2}\right]_{C}^{M}=2 \xi_{[1}^{N} \partial_{N} \xi_{2]}^{M}-\xi_{[1}^{P} \partial^{M} \xi_{2] P} .
$$

The C-bracket is the $\mathrm{O}(d, d)$ covariantization of the Courant bracket in generalized geometry [14-16].

The DFT action presented in (2.3) is invariant under the following transformations:

$$
\mathbb{S}(X) \longrightarrow \mathbb{S}^{\prime}\left(X^{\prime}\right)=\left(S^{-1}\right)^{\dagger} \mathbb{S}(X) S^{-1}, \quad \chi(X) \longrightarrow \chi\left(X^{\prime}\right)=S \chi(X)
$$

Here $S \in \operatorname{Spin}^{+}(d, d)$ and $X^{\prime}=h X$, where $h=\rho(S) \in \mathrm{SO}^{+}(d, d)$. The dilaton field is invariant. Although the DFT action would be invariant under the above transformations with a general $\operatorname{Pin}(d, d)$ element $S$, the chirality of the spinor field $\chi$ is preserved only by the $\operatorname{Spin}(d, d)$ subgroup. On the other hand, in order to preserve the self-duality constraint (2.5), the duality group should be further reduced to $\operatorname{Spin}^{+}(d, d)$. This is the reason why the actual duality group in $(2.15)$ is $\operatorname{Spin}^{+}(d, d)$. The gauge transformation rules for the generalized metric $\mathcal{H}=\rho(\mathbb{S})$ is determined by those of $\mathbb{S}$ and is as given below:

$$
\mathcal{H}(X) \longrightarrow \mathcal{H}^{\prime}\left(X^{\prime}\right)=\left(h^{-1}\right)^{T} \mathcal{H}(X) h^{-1} .
$$

\footnotetext{
${ }^{3}$ Note that, here we work locally and work with the Clifford algebra on $R^{2 d}$. A more careful analysis would require to work on the $2 d$ dimensional tangent space of the doubled manifold and discuss how the local structure can be transported to the whole doubled manifold. For a more detailed discussion, see [34].
} 
In determining the reduction anzats, we also take into account the global shift symmetry $d \rightarrow d+\rho$ of the dilaton field, which acts as a conformal rescaling on the NS-NS sector [27]. This symmetry extends to the RR sector too, provided that the spinor field $\chi(X)$ transforms as $\chi \rightarrow e^{-\rho} \chi$ [34]. Finally, the DFT Lagrangian (2.4) is also invariant under the shift of the spinor field $\chi \rightarrow \chi+\alpha$, where $\alpha$ is a constant spinor field. Although this symmetry is not respected by the gauge sector, it is still possible to take it into account in the reduction anzats, as was discussed in [34]. All symmetries considered, one ends up with the following duality twisted anzats [34]:

$$
\begin{aligned}
& \mathbb{S}(X, Y)=\left(S^{-1}\right)^{\dagger}(Y) \mathbb{S}(X) S^{-1}(Y), \\
& \chi(X, Y)=e^{-\rho(Y)} S(Y)(\chi(X)+\alpha), \\
& d(X, Y)=d(X)+\rho(Y) .
\end{aligned}
$$

Here, $X$ denote collectively the coordinates of the reduced theory. The $Y$ coordinates are the internal coordinates, which will be integrated out eventually. One can further decompose these coordinates into dual and standard coordinates as $Y=(\tilde{y}, y)$ and $X=$ $(\tilde{x}, x)$. The twist matrix $S(Y) \in \operatorname{Spin}^{+}(d, d)$ encodes the whole dependence of the fields and the gauge parameters on the internal coordinates. Obviously, the above anzats implies the following anzats in the NS-NS sector:

$$
\mathcal{H}_{M N}(X, Y)=U_{M}^{A}(Y) \mathcal{H}_{A B}(X) U^{B}{ }_{N}(Y)
$$

The duality twisted dimensional reduction of the DFT action of the NS-NS sector with the anzats (2.17), (2.18), (2.19) and (2.20) has been studied by several groups [25-27]. The resulting theory was dubbed Gauged Double Field Theory (GDFT). The duality twisted dimensional reduction of the DFT action of the RR sector with the anzats (2.17) has been recently studied by [34]. Below we present briefly the reduced actions, gage transformation rules and the consistency conditions for the reductions. For more details, see [25-27, 34].

The reduced theory is determined by the so called fluxes $f_{A B C}, \eta_{A}$, which are defined as below:

$$
\begin{aligned}
f_{A B C} & =3 \Omega_{[A B C]}, \quad \eta_{A}=\partial_{M}\left(U^{-1}\right)_{A}^{M}-2\left(U^{-1}\right)_{A}^{M} \partial_{M} \rho, \\
\Omega_{A B C} & =-\left(U^{-1}\right)_{A}^{M} \partial_{M}\left(U^{-1}\right)_{B}^{N} U_{N}^{D} \eta_{C D} .
\end{aligned}
$$

Here $U=(\rho(S))^{-1}=\rho\left(S^{-1}\right)$, where $\rho$ is the double covering homomorphism. Note that $\Omega_{A B C}$ are antisymmetric in the last two indices: $\Omega_{A B C}=-\Omega_{A C B}$. We also make the following definition

$$
f_{A}=-\partial_{M}\left(U^{-1}\right)_{A}^{M}=\Omega_{A C}^{C}
$$


The conditions for the consistency of the reduction of the NS-NS sector can be listed as below ${ }^{4}[25-27]$ :

$$
\begin{aligned}
f_{B C}^{A} \partial_{A} g(X) & =0, \\
f_{E[A B} f_{C] D}^{E} & =0, \\
\eta^{A} & =0 .
\end{aligned}
$$

In addition to the above, the weak and the strong constraint has to be imposed on the external space so that

$$
\partial_{A} \partial^{A} V(X)=0, \quad \partial_{A} V(X) \partial^{A} W(X)=0
$$

for any fields or gauge parameters $V, W$ that has dependence on the coordinates of the external space only. The last but not the least, all fluxes must be constant for the consistency of the reduced theory. This then ensures that the $Y$ dependence is completely integrated out in the reduced theory. Surprisingly, it is not necessary to impose the strong and the weak constraints in the internal space, that is, one does not need to impose

$$
\partial^{P} U_{M}^{A} \partial_{P} U_{N}^{B}, \quad \partial_{P} \partial^{P} U_{M}^{A} \text {. }
$$

Therefore, the duality twisted anzats (2.17)-(2.20) allows for a relaxation of the constraints on the total space.

The requirement of consistency of the reduction of the DFT of the RR sector brings in one more condition: the fluxes should also satisfy

$$
f_{A B C} f^{A B C}=0 .
$$

Without this extra condition the reduced action cannot be invariant under the reduced gauge transformation rules. It is known that the constraints of GDFT are in one-to-one correspondence with the constraints of half-maximal gauged supergravity [27]. We would like to remark that the extra condition (2.29) means the gauged supergravity we have obtained is a truncation of maximal supergravity [35, 36].

Once the conditions discussed above are imposed, one finds that the theory that results from the duality twisted reduction of the DFT of Type II strings is a consistent theory with the following Lagrangian for the RR sector (we discuss the NS-NS sector in the appendix. Full details can be found, for example, in [27].)

$$
L_{\mathrm{red}}=\frac{1}{4}\left\langle\not \nabla \chi(X)+\bar{\alpha}, C^{-1} \mathbb{S}(\not \nabla \chi(X)+\bar{\alpha})\right\rangle .
$$

Here, the Dirac operator $\not$ and the constant spinor field $\bar{\alpha}$ are defined as

$$
\begin{aligned}
\not & \equiv \not \partial+\frac{1}{6} f_{A B C} \psi^{A} \psi^{B} \psi^{C}+\frac{1}{2} \eta_{B} \psi^{B}, \\
\bar{\alpha} & \equiv\left(\frac{1}{6} f_{A B C} \psi^{A} \psi^{B} \psi^{C}+\frac{1}{2} \eta_{B} \psi^{B}\right) \alpha .
\end{aligned}
$$

\footnotetext{
${ }^{4}$ Despite the condition (2.26), we will be including the $\eta^{A}$ terms in the following formulas for completeness, as it might be possible to relax this condition by considering warped compactifications [25, 27]. For non-vanishing $\eta^{A}$ one also needs to impose the conditions $\eta^{A} f_{A B C}=0$ and $\eta^{A} \partial_{A} g(X)=0$ for the consistency of both the NS-NS and the RR sectors.
} 
It can be shown that the reduced Lagrangian can be rewritten as [34]

$$
L_{\mathrm{red}}=\frac{1}{4}\left\langle F(X), C^{-1} S_{g}^{-1} F(X)\right\rangle+\frac{1}{2}\left\langle F(X), C^{-1} S_{g}^{-1} \bar{\chi}_{B}\right\rangle+\frac{1}{4}\left\langle\bar{\chi}_{B}, C^{-1} S_{g}^{-1} \bar{\chi}_{B}\right\rangle,
$$

where we have defined ${ }^{5} F(X)=S_{b} \not \partial \chi(X)=e^{-B} \wedge \not \partial \chi(X)$ and $\bar{\chi}_{B}=S_{b}(\bar{\chi}+\bar{\alpha})=e^{-B} \wedge$ $(\bar{\chi}+\bar{\alpha})$, with

$$
\bar{\chi}(X)=\left(\frac{1}{6} f_{A B C} \psi^{A} \psi^{B} \psi^{C}+\frac{1}{2} \eta_{B} \psi^{B}\right) \chi(X)
$$

and $\bar{\alpha}$ is as in (2.32). If the external coordinates $X$ do not include any dual coordinates, then the above Lagrangian is equivalent to the following one

$$
L_{\mathrm{red}}=\frac{1}{4} F(X) \wedge * F(X)+\frac{1}{2} F(X) \wedge * \bar{\chi}_{B}+\frac{1}{4} \bar{\chi}_{B} \wedge * \bar{\chi}_{B},
$$

where $*$ is the Hodge duality operator with respect to the metric $g(X)$.

On the other hand, the constraint reduces to

$$
\not \nabla \chi(X)+\bar{\alpha}=-C^{-1} \mathbb{S}(\not \varnothing \chi(X)+\bar{\alpha}) .
$$

The deformed gauge transformation rules for the reduced theory in the RR sector are

$$
\begin{aligned}
& \hat{\delta}_{\hat{\xi}} \chi \equiv \delta_{\hat{\xi}} \chi+\frac{1}{4} f_{B C}^{A} \hat{\xi}_{A} \Gamma^{B} \Gamma^{C}(\chi+\bar{\alpha})+\frac{1}{2} \eta^{A} \hat{\xi}_{A}(\chi+\bar{\alpha}) \\
& \delta_{\hat{\xi}} \chi \equiv \hat{\xi}^{A} \partial_{A} \chi+\frac{1}{2} \partial_{B} \hat{\xi}_{C} \Gamma^{B} \Gamma^{C}(\chi+\bar{\alpha}) \\
& \hat{\delta}_{\hat{\lambda}} \chi=\left(\not \partial+\frac{1}{6} f_{A B C} \psi^{A} \psi^{B} \psi^{C}+\frac{1}{2} \eta_{B} \psi^{B}\right) \hat{\lambda} .
\end{aligned}
$$

Here, we have defined $\xi^{M}(X, Y)=\left(U^{-1}\right)^{M} \hat{\xi}^{A}(X), \lambda(X, Y)=e^{-\rho(Y)} S(Y) \hat{\lambda}(X), \delta_{\xi} \chi=$ $S(Y)\left(\hat{\delta}_{\hat{\xi}} \chi\right)$, and $\delta_{\xi} \lambda=S(Y)\left(\hat{\delta}_{\hat{\lambda}} \chi\right)$.

It was shown in [34] that the reduced Lagrangian (2.30) is invariant under the deformed gauge transformations with parameter $\hat{\lambda}$, only when the Dirac operator $\not$ is nilpotent. It was also shown that the Dirac operator is nilpotent if and only if the extra condition (2.29) is satisfied. On the other hand, the constraints (2.24)-(2.27) required for the consistency of the NS-NS sector are sufficient to ensure the gauge invariance of (2.30) under gauge transformations with parameter $\hat{\xi}$ and they also suffice for the deformed gauge transformations close to form an algebra with respect to the deformed C-bracket [34].

We would like to note that in finding the reduced Lagrangian and the gauge transformations, the following identity, which follows from the fact that the Lie algebras of $\mathrm{SO}(d, d)$ and $\operatorname{Spin}(d, d)$ are isomorphic plays a crucial role [34]:

$$
\Gamma^{A}\left(U^{-1}\right)^{M}{ }_{A} S^{-1} \partial_{M} S=\frac{1}{12} f_{A B C} \Gamma^{A} \Gamma^{B} \Gamma^{C}-\frac{1}{2} f_{B} \Gamma^{B} .
$$

\footnotetext{
${ }^{5}$ Here, $S_{b}$ is the spin group element whose spinorial action on polyforms corresponds to $B$ field shifts, as we will discuss in more detail in section 3.1.
} 


\section{Double field theory of massive Type IIA theory}

The DFT action reduces to the standard Type II supergravity action in the supergravity frame $\tilde{\partial}^{i}=0$. In the $\mathrm{RR}$ sector, the action reduces to the action of massless Type II theory written in the democratic formulation [42, 43], as was shown explicitly in [17]. Here, one should fix the chirality of the spinor field $\chi$ at the outset. Positive chirality gives rise to Type IIB theory, whereas negative chirality yields Type IIA theory. Conversely, if one writes the democratic formulation of the RR action in terms of the Mukai pairing, which is a $\operatorname{Spin}(n, n)$ invariant bilinear form on the space of differential forms, then it is easily seen that it extends naturally to the action (2.4) of [21], as was discussed in [34].

The Lagrangians for the democratic formulation of Type II theories can be written as [34]:

$$
L=\frac{1}{4}\left\langle F^{ \pm}, C^{-1} S_{g}^{-1} F^{ \pm}\right\rangle .
$$

Here, $F^{+}$and $F^{-}$are polyforms consisting of even and odd degree forms, respectively. The choice with $F^{+}$gives the Lagrangian for the Type IIA theory, whereas the choice with $F^{-}$ gives the Lagrangian for the Type IIB theory. $C$ is the charge conjugation matrix and $S_{g}^{-1}$ is the spin representative of the inverse metric $g^{-1}$ [34]. The spinorial action of $C^{-1} S_{g}^{-1}$ on a spinor field $\varphi$ amounts to taking the Hodge dual of it with respect to the metric $g$, when $\varphi$ is regarded as a differential form $[17,34]$. Now, let $\chi$ be the chiral spinor field, which encodes the (modified) RR gauge potentials and their Hodge duals. It was shown in [34] that (3.1) can be rewritten as

$$
L=\frac{1}{4}\left\langle\psi^{i} \partial_{i} \chi, C^{-1} \mathbb{S} \psi^{i} \partial_{i} \chi\right\rangle .
$$

The crucial point to note here is that the spinor field $\chi$, as a differential form, is related to $F^{ \pm}$that appears in the democratic action in the following way: $F^{ \pm}=S_{b} \psi^{i} \partial_{i} \chi$, where $F^{+}$corresponds to the negative chirality and $F^{-}$corresponds to the positive chirality of $\chi$. Then, we have (dropping the superscripts \pm for convenience):

$$
L=\frac{1}{4}\left\langle F, C^{-1} S_{g}^{-1} F\right\rangle=\frac{1}{4}\left\langle S_{b} \psi^{i} \partial_{i} \chi, C^{-1} S_{g}^{-1} S_{b} \psi^{i} \partial_{i} \chi\right\rangle .
$$

The right hand side of this equation is indeed equivalent to (3.2), as one has $\mathbb{S}=S_{b}^{\dagger} S_{g}^{-1} S_{b}$, where $S_{b}^{\dagger} \equiv C S_{b}^{-1} C^{-1}[17,34]$. Also, the Mukai pairing is invariant under the action of $S_{b}$, that is, $\left\langle S_{b} \phi, S_{b} \phi\right\rangle=\langle\phi, \phi\rangle$ for any spinor field $\phi[16]$.

The action (3.2) has to be supplemented by the following self-duality equation:

$$
\not \partial \chi=-C^{-1} \mathbb{S} \not \partial \chi
$$

This condition is equivalent to the duality condition [21]

$$
F_{10-p}=(-1)^{\left[\frac{p-1}{2}\right]} * F_{p}
$$

Here $F_{p}$ are components of the polyform $F$ and $\left[\frac{p-1}{2}\right]$ is the first integer greater than or equal to $\frac{p-1}{2}$. When one imposes this relation to the field equations derived from the action 
in the democratic formulation, the field equations for the higher degree fields (which come from the Hodge duals of the RR potentials) become equivalent to the Bianchi identities of the RR potential fields. It is straightforward to extend this Lagrangian to its DFT version. One just allows the fields $\mathbb{S}$ and $\chi$ to depend on the winding coordinates as well as the standard ones so that $\chi=\chi(x, \tilde{x})$ and $\mathbb{S}=\mathbb{S}(x, \tilde{x})$. Then the Dirac operator $\psi^{i} \partial_{i}$ should also be extended to $\not \partial$ in (2.7) and the Lagrangian becomes

$$
L=\frac{1}{4}\left\langle\not \partial \chi, C^{-1} \mathbb{S} \not \partial \chi\right\rangle .
$$

The duality relation (3.4) also extends trivially. Both the action and the self-duality relation are manifestly $\operatorname{Spin}(d, d)$ invariant, as the Mukai pairing is itself $\operatorname{Spin}(d, d)$ invariant. However, it is crucial to include the dual coordinates, as $\chi \rightarrow S \chi$ implies $\not \partial \chi \rightarrow S \not \partial \chi$ only when the dual coordinates are introduced [34]. ${ }^{6}$

The massive IIA action also includes a 0 -form field strength $F_{0}$ [44]. In order to take this extra 0-form field strength into account, one has to modify [21, 43]

$$
F \longrightarrow F+e^{-B} \wedge F_{0}
$$

The Hodge duality relation between $F_{0}$ and $F_{10}$, which follows from (3.5), along with the field equation $d * F_{10}=0$ implies that $d F_{0}=0$, that is, $F_{0}=m$ is constant. The corresponding action is equivalent to the Romans massive IIA supergravity action, again in the above sense: varying the democratic action and imposing the duality constraint (3.4), one obtains the Bianchi identities and the field equations for the p-form fields in Romans' theory. Note that, regarding $F_{0}$ as a 0 -form field strength is somewhat artificial, as the gauge potential that gives rise to it would have to be a (-1)-form field. In spite of this, such a (-1)-form field was introduced formally in [45]. More recently, the existence of such a (-1)-form field was considered in [21] within the context of DFT, where they interpreted it as a 1-form field, which depends on the dual winding coordinates of DFT. The anzats [21] chooses for the spinor field $\chi$ in order to induce such a mass deformation is as follows:

$$
\chi(x, \tilde{x})=\chi_{0}(x)+\chi_{1}(\tilde{x}),
$$

where the only non-vanishing component of the spinor field $\chi_{1}(\tilde{x})$ is its 1-form part, and it depends on one of the dual coordinates $\tilde{x}_{1}$ linearly: $\chi_{1}(\tilde{x})=m \tilde{x}_{1}$. The dependence on the dual coordinate $\tilde{x}$, being only linear, drops out when the anzats (3.8) is plugged into $\not \partial \chi$ yielding $\not \partial \chi(x, \tilde{x})=\psi^{i} \partial_{i} \chi_{0}(x)+\psi_{i} \tilde{\partial}^{i} \chi_{1}(\tilde{x})=\psi^{i} \partial_{i} \chi_{0}(x)+m$. Then, $S_{b} \not \partial \chi(x, \tilde{x}) \rightarrow$ $S_{b}\left(\psi^{i} \partial_{i} \chi_{0}(x)+m\right)=F+e^{-B} \wedge F_{0}$, where, as above, we identify $F(x)$ with $S_{b}\left(\psi^{i} \partial_{i} \chi_{0}(x)\right)$ and $F_{0}$ with $m$. As a result, the linear dependence of the 1-form part of the spinor field $\chi$ on the dual coordinate $\tilde{x}$ indeed induces the mass deformation desired. Note that the anzats (3.8) of [21] violates the strong constraint, although it respects the weak constraint. Also note that the gauge transformation rule (2.12) depends on $\chi$ explicitly (unlike the DFT action, which only depends on derivatives of $\chi$ ) and hence the linear dependence on $\tilde{x}$ does not

\footnotetext{
${ }^{6}$ To demonstrate invariance under $\operatorname{Spin}(d, d)$, one should also use the fact that $C^{-1} \mathbb{S} S= \pm S C^{-1} \mathbb{S}$ for any $S \in \operatorname{Spin}(d, d)$. For details, see [34].
} 
automatically drop from the gauge algebra. Therefore, the standard gauge transformation rules for $\chi$ that we presented in the previous sections have to be reformulated, so that the closure of the gauge algebra requires only the weak constraint [21].

In this paper, we will introduce a different anzats, which will also induce a deformation of the type above. More precisely, we will consider whether an anzats of the form (2.18) for the spinor field $\chi$ might give rise to a massive deformation of Type IIA theory. We will see that this is indeed possible for certain choices of the twist matrix $U \in \mathrm{SO}^{+}(d, d)$ and the corresponding $S \in \operatorname{Spin}^{+}(d, d)$. Interestingly, for one choice of the twist matrix, our anzats violates both the strong and the weak constraints. The consistency of our anzats owes to the fact that the strong and the weak constraints are violated only in the internal space. The possibility of relaxation of the constraints of DFT in the NS-NS sector through duality twisted reduction anzatse was first noticed in $[25,26]$ and was established rigorously in [27], as we discussed in section 2. The consistency of such reductions in the RR sector was studied in [34]. We also would like to mention that, our anzats does not require reformulation of the gauge transformation rules in the $\mathrm{RR}$ sector as in [21], and we have full control over the effects of our anzats in the NS-NS sector.

As we saw in the previous section, the deformation induced by a duality twisted anzats of the form (2.17), (2.18) is determined completely by the fluxes $f_{A B C}$ and $\eta_{A}$. Therefore, we would like to compute these fluxes first, in order to see the form of the twist matrices that might give rise to massive deformations of Type IIA theory.

\section{$3.1 \quad$ Fluxes}

Recall that the twist element $S(Y)$ must be in the identity component of the spin group: $S(Y) \in \operatorname{Spin}^{+}(d, d)$. Let us briefly recall the classification of elements of $\operatorname{Spin}^{+}(d, d)$ and their spinorial action on spinor fields $[17,34]$. The identity component $\operatorname{Spin}^{+}(d, d)$ of the spinor group $\operatorname{Spin}(d, d)$ is obtained by exponentiating the generators of the Lie algebra $\operatorname{so}(d, d) \cong \operatorname{spin}(d, d)$ in the spin representation. This gives the elements $S_{B}, S_{\beta}, S_{A}$ below, which act on a spinor field $\alpha$ as follows:

$$
\begin{aligned}
& S_{B}: \quad \alpha \longmapsto e^{-B} \wedge \alpha=\left(1-B+\frac{1}{2} B \wedge B-\ldots\right) \wedge \alpha, \\
& S_{\beta}: \quad \alpha \longmapsto e^{\beta} \alpha=\left(1+i_{\beta}+\frac{1}{2} i_{\beta}^{2}+\cdots\right) \alpha, \\
& S_{A}: \quad \alpha \longmapsto \frac{1}{\sqrt{\operatorname{det} R}}\left(e^{A}\right)^{*} \alpha .
\end{aligned}
$$

Here $B=\frac{1}{2} B_{k l} \psi^{k} \wedge \psi^{l}$, and $\beta=\frac{1}{2} \beta^{k l} \psi_{k} \wedge \psi_{l}$. Also, $i_{\beta} \alpha=\frac{1}{2} \beta^{i j} i_{\psi_{i}}\left(i_{\psi_{j}} \alpha\right)$ and $R^{*} \alpha=$ $R_{j}{ }^{i} \psi^{j} \wedge i_{\psi_{i}} \alpha$, with $R=e^{A}$, which is the usual action of $\mathrm{GL}^{+}(d)$ on forms, where $\mathrm{GL}^{+}(d)$ is the space of (orientation preserving) linear transformations of strictly positive determinant. Here, we have identified the spinor fields with (not necessarily homogenous) differential forms (or with polyforms, as is commonly called in physics literature), as in $(2.10)[16,17]$. 
Under the double covering homomorphism $\rho: \operatorname{Spin}(d, d) \longrightarrow \mathrm{SO}(d, d)$, these elements project onto the $\mathrm{SO}^{+}(d, d)$ elements $h_{B}, h_{\beta}$ and $h_{A}$, respectively, where

$$
\begin{array}{rlr}
h_{B}=\left(\begin{array}{cc}
1 & -B \\
0 & 1
\end{array}\right), & B^{T}=-B, \\
h_{\beta}=\left(\begin{array}{ll}
1 & 0 \\
\beta & 1
\end{array}\right), & \beta^{T}=-\beta \\
h_{A}=\left(\begin{array}{cc}
e^{A} & 0 \\
0 & \left(e^{-A}\right)^{T}
\end{array}\right), &
\end{array}
$$

The corresponding elements $S$ and $h$ satisfy

$$
\Gamma_{N} h_{M}^{N}=S \Gamma_{M} S^{-1}, \quad\left(h^{-1}\right)^{M}{ }_{N}^{N}=S \Gamma^{M} S^{-1} .
$$

When we choose the twist matrix $S$ as one of $S=S_{B}(y, \tilde{y}), \quad S=S_{A}(y, \tilde{y})$ or $S=$ $S_{\beta}(y, \tilde{y})$, we introduce the dependence on the coordinates $y, \tilde{y}$ through the parameters $B=B(y, \tilde{y}), A=A(y, \tilde{y}), \beta=\beta(y, \tilde{y})$. In order to see how the DFT action is deformed in the RR sector (as well as the NS-NS sector), it is enough to calculate $f_{A B C}$ and $\eta_{A}$ in (2.21) for the corresponding $\mathrm{SO}^{+}(d, d)$ matrices $U_{B}^{-1}, U_{A}^{-1}, U_{\beta}^{-1}$, as we discussed in section 2 .

Let us consider the general case

$$
U^{-1}=h_{\beta} h_{A} h_{B}=\left(\begin{array}{cc}
e^{A} & e^{A} B \\
\beta e^{A} & \beta e^{A} B+\left(e^{-A}\right)^{T}
\end{array}\right) .
$$

Computation of the corresponding fluxes $f_{a b c}, f_{b c}^{a}, f_{c}^{a b}, f^{a b c},{ }^{7}$ has been carried out by various groups $[26,28,31,46,47]$. It is common to refer to them as the H-flux, geometric flux, Q-flux and R-flux [48] (in the order they have been listed above), and we will follow the same terminology.

If we introduce our duality twist through the $\operatorname{Spin}^{+}(d, d)$ element $S=S_{\beta} \cdot S_{A} \cdot S_{B}$, then the fluxes corresponding to $U^{-1}=\rho\left(S_{\beta} \cdot S_{A} \cdot S_{B}\right)=\rho\left(S_{\beta}\right) \rho\left(S_{A}\right) \rho\left(S_{B}\right)=h_{\beta} h_{A} h_{B}$ in (3.16) is computed from the below: ${ }^{8}$

$$
\begin{aligned}
\Omega_{a b c}= & R_{a}^{i}\left(\partial_{i}+R_{j}^{d} R_{i}^{e} B_{d e} \tilde{\partial}^{j}\right) B_{b c}+\Gamma_{a[b}{ }^{d} B_{c] d}+B_{e a} \Gamma_{[b}^{e d} B_{c] d} \\
& +\beta^{k m} R_{k}{ }^{d} R_{m}^{f} B_{a d}\left(R_{f}^{i} \partial_{i} B_{b c}+\Gamma_{f[b}{ }^{e} B_{c] e}\right)+R_{a}^{i} R_{k}{ }^{e} R_{j}^{f} B_{e b} B_{j f} \partial_{i} \beta^{j k} \\
& +R_{a}^{i} R_{k}{ }^{e} R_{j}{ }^{f} B_{e b} B_{d a}\left(\tilde{\partial}^{i}+\beta^{l i} \partial_{l}\right) \beta^{j k}
\end{aligned}
$$

\footnotetext{
${ }^{7}$ Note that, due to complete antisymmetry of $f_{A B C}$ in its indices, these are the only independent blocks out of the 8 possible combinations.

${ }^{8}$ Note that in the aforementioned references, where they have also computed the fluxes, the twist matrix is of the form

$$
U^{-1}=h_{B} h_{A} h_{\beta}=\left(\begin{array}{cc}
B\left(e^{-A}\right)^{T} \beta+e^{A} & B\left(e^{-A}\right)^{T} \\
\left(e^{-A}\right)^{T} \beta & \left(e^{-A}\right)^{T}
\end{array}\right) .
$$

This brings in some differences in the computation of fluxes. However, our results agree with theirs when $\beta=0$ and/or $B=0$.
} 


$$
\begin{aligned}
\Omega_{b c}^{a}= & R_{l}{ }^{a} R_{m}{ }^{e} \beta^{m l} \Gamma_{e[b}{ }^{d} B_{c] d}+\Gamma^{a e}{ }_{[b} B_{c] e}+R_{i}{ }^{a}\left(\tilde{\partial}^{i}+\beta^{l i} \partial_{l}\right) B_{b c} \\
& -R_{i}{ }^{a} R_{k}{ }^{d} R_{j}{ }^{e} B_{d b} B_{e c}\left(\tilde{\partial}^{i}+\beta^{l i} \partial_{l}\right) \beta^{j k}, \\
\Omega_{a b}{ }^{c}= & -\Gamma_{a b}{ }^{c}+R_{k}{ }^{b} R_{m}{ }^{e} \beta^{m k} \Gamma_{e b}{ }^{c}+B_{a d} \Gamma^{d c}{ }_{b}-R^{i}{ }_{a} R_{k}{ }^{d} R_{m}{ }^{c} B_{d b}\left(\partial_{i}+R_{j}{ }^{e} R_{i}{ }^{f} B_{e f} \tilde{\partial}^{j}\right) \beta^{m k}, \\
\Omega_{a c}{ }^{b}= & -\Omega_{a c}{ }^{b} \\
\Omega^{a b}{ }_{c}= & \Gamma^{a b}{ }_{c}-R_{l}{ }^{a} R_{m}{ }^{d} B^{l m} \Gamma_{d c}{ }^{b}+R_{i}{ }^{a} R_{k}{ }^{b} R_{j}{ }^{d} B_{c d}\left(\tilde{\partial}^{i}+\beta^{l i} \partial_{l}\right) \beta^{j k}, \\
\Omega_{a}{ }^{b c}= & R^{i}{ }_{a} R_{j}{ }^{b} R_{k}{ }^{c} \partial_{i} \beta^{j k}+R_{i}{ }^{d} R_{j}{ }^{c} R_{k}{ }^{b} B_{a d}\left(\tilde{\partial}^{i}+\beta^{l i} \partial_{l}\right) \beta^{j k}, \\
\Omega^{a}{ }_{b}{ }^{c}= & -\Omega^{a c}{ }_{b} \\
\Omega^{a b c}= & R_{i}{ }^{a} R_{j}{ }^{b} R_{k}{ }^{c}\left(\tilde{\partial}^{i}+\beta^{l i} \partial_{l}\right) \beta^{j k},
\end{aligned}
$$

Here $R_{i}{ }^{a}=\left(e^{A}\right)_{i}{ }^{a}$ and $R_{a}^{i}=\left(\left(e^{-A}\right)^{T}\right)^{i}{ }_{a}$. Also we have defined

$$
\begin{aligned}
\Gamma_{a b}{ }^{c} & =-R^{i}{ }_{a} \partial_{i} R_{j}{ }^{c} R^{j}{ }_{b} \\
\Gamma^{a b}{ }_{c} & =-R_{i}{ }^{a} \tilde{\partial}^{i} R_{j}{ }^{b} R^{j}{ }_{c} .
\end{aligned}
$$

Now one can find the fluxes from (2.21). For example, the Q-flux is computed as

$$
f_{a}^{b c}=\Omega_{a}^{b c}+\Omega_{a}^{b c}+\Omega_{a}^{c},
$$

and similarly for the other fluxes.

\subsection{Determining the twist element that gives rise to massive IIA}

The first and the obvious condition on the duality twisted anzats that will yield a massive deformation of Type IIA theory is that the external coordinates $X$ should not include any dual coordinates. In order to determine the twist matrix $\mathrm{U}(Y)$ and the corresponding $S(Y)$, let us recall that the whole deformation induced by the duality twisted anzats is encoded in $\bar{\chi}$ and $\bar{\alpha}$ in (2.34) and (2.32), respectively. Therefore, we are interested in the twists for which these spinor fields include a 0 -form part. Recall that $\psi^{i}$ act on a spinor field $\phi$ (regarded as a differential form) by wedge product, whereas $\psi_{i}$ acts on it by contraction, see (2.10) and (2.11). Therefore, the only way to obtain a 0-form field through the action of $\frac{1}{6} f_{A B C} \psi^{A} \psi^{B} \psi^{C}$ is ${ }^{9}$ to act by $\psi_{i}$ with lower indices only. Therefore, we are interested in the fluxes $f_{A B C}$, which have either 3 upper indices $f^{a b c}$ (R-flux), or 2 upper indices and 1 lower index of the form $f_{a}{ }^{b a}$ (trace of the Q-flux). ${ }^{10}$ As we are not interested in the geometric flux and the H-flux, we can assume that $\partial_{m} R_{a}^{i}=0$, that is, the components of the matrix $A$ do not depend on any of the standard coordinates $y$, and we also take $B=0$. This immediately ensures that the geometric flux and the H-flux vanish, as can be seen easily from the flux formulas presented above. The expressions for the remaining flux components also simplify significantly. We have

$$
\begin{aligned}
& \Omega_{c}^{a b}=-R_{i}{ }^{a} R^{j}{ }_{c} \tilde{\partial}^{i} R_{j}^{b} \\
& \Omega_{a}^{b c}=R_{a}^{i} R_{j} R_{k}{ }^{c} \partial_{i} \beta^{j k} \\
& \Omega^{a b c}=R_{i}{ }^{a} R_{j}{ }^{b} R_{k}{ }^{c}\left(\tilde{\partial}^{i}+\beta^{l i} \partial_{l}\right) \beta^{j k} .
\end{aligned}
$$

\footnotetext{
${ }^{9}$ From now on we are taking $\eta^{A}=0$ as is necessary for the gauge invariance of the reduced action.

${ }^{10}$ Note that this is different from $f^{b}$. We have $f_{a}{ }^{b a}=\Omega_{a}^{b a}+\Omega^{b a}{ }_{a}+\Omega^{a}{ }_{a}^{b}$, whereas $f^{b}=\Omega_{A}^{b A}=\Omega_{a}^{b a}+\Omega^{a b}{ }_{a}=$ $\Omega_{a}^{b a}-\Omega_{a}^{a b}$.
} 


\subsubsection{Twists with $S(y, \tilde{y})=S_{A}(\tilde{y}), \beta=0$}

Let us take the $\operatorname{Spin}^{+}(10,10)$ twist matrix $S(y, \tilde{y})=S_{A}(\tilde{y})$ as in (3.11). We simplify matters by taking $A$ to depend on one of the dual coordinates only, which we call $\tilde{x}_{1},{ }^{11}$ and we also take this dependence to be linear. Let $A\left(\tilde{x}_{1}\right)=A \tilde{x}_{1}$, where $A$ is in $g l(10)$. Then $\left(U^{-1}\right)=h_{A}=\rho\left(S_{A}\right)$, where $h_{A}$ is as in (3.14) with $e^{A\left(\tilde{x}_{1}\right)}=e^{A \tilde{x}_{1}}$. We calculate $\Omega_{A B C}$ from (3.23) and find

$$
\Omega_{c}^{a b}=-\left(e^{A \tilde{x}_{1}}\right)_{1}{ }^{a} A_{c}{ }^{b}, \quad \Omega^{a}{ }_{b}^{c}=+\left(e^{A \tilde{x}_{1}}\right)_{1}{ }^{a} A_{b}{ }^{c}, \quad \Omega_{a}^{b c}=0 .
$$

This gives the following fluxes

$$
f_{a}^{b c}=\Omega_{a}^{b c}+\Omega^{b c}{ }_{a}+\Omega_{a}^{c}{ }^{b}=\Omega^{[b c]}{ }_{a}=-R_{i}{ }^{[b} R^{j}{ }_{a} \tilde{\partial}^{i} R_{j}^{c]}=-\left(e^{A \tilde{x}_{1}}\right)_{1}{ }^{[b} A_{a}^{c]} .
$$

Also note that $f_{a}=\Omega^{c}{ }_{a c}+\Omega_{c a}{ }^{c}=0$ and $f^{a}=\Omega^{c a}{ }_{c}+\Omega_{c}{ }^{a c}=\Omega^{c a}{ }_{c}=-\left(e^{A \tilde{x}_{1}} A\right)_{1}{ }^{a}=-\tilde{\partial}^{1} R_{1}{ }^{a}=$ $-\tilde{\partial}^{1}\left(U^{-1}\right)_{1}^{a}$, as it has to be.

Consider the following simple choice of the twist matrix $U$, where $A$ induces an $\operatorname{SL}(2, \mathrm{R})$ twist in the parabolic conjugacy class along the 1st and 2nd directions so that the only non-zero component of the matrix $A$ is $A_{1}{ }^{2}=-m$. Then we have $R_{i}{ }^{a}=\left(e^{A \tilde{x}_{1}}\right)_{i}{ }^{a}=$ $\delta_{i}{ }^{a}-m \tilde{x}_{1} \delta_{i}{ }^{1} \delta_{2}{ }^{a}$. This then gives us $f_{1}{ }^{12}=m$, and $f^{2}=m$. The non-vanishing flux $f^{2}$ contributes to $\eta^{2}$ as can be seen from (2.21) and (2.23). This should be compensated by a non-trivial dilaton anzats (2.19) with non-constant $\rho$. We will discuss this in more detail shortly. For the time being, let us assume that $\rho$ has been chosen so as to yield vanishing $\eta^{2}$, as is required for gauge invariance. Then we have ${ }^{12}$

$$
\bar{\chi}=\left(m \psi_{1} \psi_{2} \psi^{1}+\frac{1}{2} m \psi_{2}\right) \chi, \quad \text { and } \quad \bar{\alpha}=\left(m \psi_{1} \psi_{2} \psi^{1}+\frac{1}{2} m \psi_{2}\right) \alpha .
$$

Let us remind the reader, once again, that the spinorial action of the Clifford algebra elements $\psi_{1}$ and $\psi_{2}$ on the spinor field $\chi$ (regarded as a differential form) is by contraction along the $x^{1}$ and $x^{2}$ directions, respectively and $\psi^{1}$ acts by wedge product. Therefore, this deformation term can give rise to a constant 0 -form in the resulting theory, when we choose the constant spinor field $\alpha$ appropriately. For example, if we choose the spinor field $\alpha$ such that its 1-form part has non-zero component along $x^{2}$ direction, with coefficient 1 and has no other p-form parts that have a non-vanishing component along this direction, then we have $\bar{\alpha}=-\frac{1}{2} m$, which is a constant 0 -form. It is also possible to induce a non-constant 0 -form field through the 1-form part of the spinor field $\chi$. On the other hand, for certain cases, for instance when $\chi$ has no p-form components with non-vanishing coefficients along the $x^{2}$ direction, we will just have $\not \chi(X)=\not \partial \chi(X)$, as we will have $\bar{\chi}=0$.

Now that we have seen that our choice of anzats can give rise to a constant (and also non-constant) 0-form field, we now would like to discuss the non-trivial reduction anzats for the dilaton field. Such non-trivial anzats is needed in order to make sure that $\eta^{2}=0$

\footnotetext{
${ }^{11}$ In other words, our only $Y$ coordinate is $\tilde{x}_{1}$. Recall that we have already excluded all the dual coordinates from the external $X$ coordinates.

${ }^{12} \mathrm{Had}$ we had a trivial dilaton anzats (2.19) with constant $\rho$ so that $\eta^{2}=-f^{2}=-m$, we would have had $\bar{\chi}=m \psi_{1} \psi_{2} \psi^{1} \chi, \quad$ and $\quad \bar{\alpha}=m \psi_{1} \psi_{2} \psi^{1} \alpha$.
} 
in the presence of non-zero $f^{2}$, as was discussed in the previous paragraph. Looking at the definition of $\eta_{A}$ in (2.21), we see that the equation $\eta_{A}=0$ is equivalent to

$$
\partial_{M}\left(U^{-1}\right)_{A}^{M}-2\left(U^{-1}\right)_{A}^{M} \partial_{M} \lambda=0 .
$$

Since we have $\left(U^{-1}\right)_{i a}=\left(U^{-1}\right)^{i a}=0$ and $\partial_{i}\left(U^{-1}\right)^{i}{ }_{a}=0$ for our choice of the twist matrix, the equation (3.27) gives us the following two sets of coupled equations

$$
\begin{aligned}
\left(U^{-1}\right)^{i}{ }_{a} \partial_{i} \lambda & =0 \\
\tilde{\partial}^{1}\left(U^{-1}\right)_{1}{ }^{a}-2\left(U^{-1}\right)_{i}{ }^{a} \tilde{\partial}^{i} \lambda & =0 .
\end{aligned}
$$

It is easily checked that

$$
\lambda=-\frac{m \tilde{x}_{2}}{2}
$$

solves these equations. Therefore, the anzats for the generalized dilaton is

$$
d(X, Y)=d(X)-\frac{m \tilde{x}_{2}}{2} .
$$

This ensures that the consistency condition (2.26) is satisfied. We find this dilaton anzats particularly interesting, given that reductions with non-trivial dilaton anzats have received some interest recently, in an attempt to understand the recently discovered generalized supergravity equations [37] within DFT [38-40]. On the other hand, the condition of constancy of $f_{A B C}$ and the Jacobi identity (2.25) are trivially satisfied. The only other condition we have to check is whether (2.24) is satisfied or not. In our case, imposing this condition immediately implies that all fields in the GDFT, including the generalized metric and the dilaton, must be independent of the coordinate $x^{1}$ and $x^{2}$ and the theory is effectively a 8-dimensional theory.

The fact that our theory is effectively 8-dimensional is perhaps not very surprising, when one recalls the work of [49], which explains how massive Type II string theory arises from M-theory. Here, one compactifies M-theory on a torus bundle B(A,R) over a circle $S^{1}$ of radius $R$, also taking the zero volume limit $A \rightarrow 0$. The fibers of the bundle is a 2-torus $T^{2}$ and its modulus depends on the coordinates of the circle $S^{1}$, where the mass parameter $m$ results from this dependence and as such becomes a measure of the non-triviality of the bundle. Then, when $m=0$, this gives a compactification on a trivial torus bundle over a circle, that is a 3 -torus, in the limit in which the volume of the 3 -torus shrinks to zero. Therefore, the description of massive string theory in [49] can be understood as a deformation of an effectively 8-dimensional theory.

Note that due to non-vanishing $f_{1}{ }^{12}$, the NS-NS sector is also deformed. We give more details about the NS-NS sector in the appendix A.

\subsubsection{Twists with $S(y, \tilde{y})=S_{\beta}(\tilde{y}), A=0$}

Let us now consider the duality twisted reduction of the theory by the $\operatorname{Spin}^{+}(10,10)$ matrix $S=S_{\beta}(\tilde{y})$, where $S_{\beta}$ is as in $(2.38)$ with $\beta=\beta(\tilde{y})$. Then the corresponding $\mathrm{SO}^{+}(10,10)$ 
matrix is $U=h_{\beta}$, where $h_{\beta}$ is as in (2.35). Repeating the calculation above we find that the only non-zero components of $\Omega$ are:

$$
\Omega^{1 b c}=\tilde{\partial}^{1} \beta^{b c}
$$

One immediately sees that the non-zero components of $\beta^{1 j}$ will not bring any non-zero contribution to $f^{1 b c}$, so without loss of generality we can take $\beta^{1 j}=0$. Also, $f^{1 b c}$ are constant only if $\beta$ is linear in $\tilde{x}$. For simplicity, we take the only non-zero components of $\beta$ to be $\beta^{23}=-\beta^{32}=m \tilde{x}_{1}$. Then we have

$$
f^{123}=f^{231}=f^{312}=m .
$$

This then gives

$$
\bar{\chi}=m \psi_{1} \psi_{2} \psi_{3} \chi \quad \text { and } \quad \bar{\alpha}=m \psi_{1} \psi_{2} \psi_{3} \alpha .
$$

This leads to a constant 0 -form (and hence a mass deformation), if we choose $\alpha$ such that the integral of the 3 -form part of it over the 3 -cycle along the directions $x^{1} x^{2} x^{3}$ is non-vanishing (and constant as $\alpha$ is a constant spinor field to begin with). It is also possible to introduce a non-constant 0 -form field through contractions of the 3 -form part of the spinor field $\chi$. On the other hand, we may also have $\bar{\chi}=0$ and hence $\not \varnothing \chi(X)=\not \partial \chi(X)$ when, for example, the 3 -form part of $\chi$ has no non-vanishing component along any of these 3 directions. Note that the resulting theory is effectively 7-dimensional, as the constraint $f^{A B C} \partial_{A} g(X)=0$ implies that no fields in the theory is allowed to depend on the coordinates $x^{1}, x^{2}, x^{3}$. For details on the deformation of the NS-NS sector, see appendix A.

\subsubsection{Mixed twists $S(y, \tilde{y})=S_{\beta}(y) . S_{A}(\tilde{y})$}

In the previous subsection 3.2.1, we introduced a twist which produces non-zero flux $f_{1}^{12}$ along with non-vanishing $f^{2}$. Since $f^{2}$ contributes to $\eta^{2}$, which is required to be zero, we had to cancel $f^{2}$ via dependence of the dilaton field on one of the dual coordinates. In this section, we will consider a new type of twist, which we will dub "the mixed twist", as it will involve both the standard coordinates $x$ and the dual coordinates $\tilde{x}$. This new twist will be of the form

$$
S=S(y, \tilde{y})=S_{\beta}(y) \cdot S_{A}(\tilde{y}),
$$

where $S_{A}$ is as in subsection 3.2.1. Therefore, we will have non-vanishing $f_{1}^{12}$ and $f^{2}$ flux, as before. This time, the non-zero $f^{2}$ flux will be cancelled not by a non-trivial dilaton anzats, but by the contribution of $S_{\beta}$ in (3.35). Choosing $S_{\beta}(y)$ such that the only non-vanishing component of $\beta$ in (3.16) is $\beta^{12}=m x^{1}$ does indeed yield the desired configuration. One can compute from (3.23) that the only non-vanishing components of $\Omega$ are

$$
\begin{array}{ll}
\Omega_{1}^{12}=-\Omega_{1}^{1}{ }_{1}{ }^{2}=m, & \Omega^{22}{ }_{1}=-\Omega^{2}{ }_{1}{ }^{2}=-m^{2} \tilde{x}^{1}, \\
\Omega_{1}{ }^{12}=-\Omega_{1}{ }^{21}=m, & \Omega^{212}=-\Omega^{221}=m^{2} \tilde{x}^{1} .
\end{array}
$$

They combine to give the flux combination exactly of the form we are seeking for: $f_{1}{ }^{12}=2 \mathrm{~m}$ and $\eta^{2}=f^{2}=0$. Then the deformation in the RR sector is exactly as in subsection 3.2.1 (except for the insignificant difference that here in this section we have $2 m$ rather than $m$ ). 
An appropriate choice of $\alpha$ induces a 0 -form and hence a mass deformation and consistency requires that the fields in the resulting GDFT depend only on the standard coordinates $\left\{x^{3}, \ldots, x^{10}\right\}$.

Note that the twists we have considered so far all involve 10 or less coordinates on the total space: the 10 coordinates $(Y, X)=\left(\left\{\tilde{x}_{1}, \tilde{x}_{2}\right\},\left\{x^{3}, \ldots, x^{10}\right\}\right)$ in Case 1 ; the 8 coordinates $(Y, X)=\left(\left\{\tilde{x}_{1}\right\},\left\{x^{4}, \ldots, x^{10}\right\}\right)$ in Case 2 and the 10 coordinates $(Y, X)=$ $\left(\left\{\tilde{x}_{1}, x^{1}\right\},\left\{x^{3}, \ldots, x^{10}\right\}\right)$ for the last case. It is also possible to come up with a configuration, which depends on a total of more than 10 coordinates on the total space and yet reproduces the same gauging. Consider the twist (3.35) again, where $S_{A}$ is again as in section 3.2.1, and $S_{\beta}$ is as in this section but this time with $\beta^{12}=2 m x^{1}$. Now we have

$$
\begin{array}{ll}
\Omega^{12}{ }_{1}=-\Omega^{1}{ }_{1}{ }^{2}=m, & \Omega^{22}{ }_{1}=-\Omega^{2}{ }_{1}{ }^{2}=-m^{2} \tilde{x}^{1}, \\
\Omega_{1}{ }^{12}=-\Omega_{1}{ }^{21}=2 m, & \Omega^{212}=-\Omega^{221}=4 m^{2} \tilde{x}^{1} .
\end{array}
$$

They combine to give the flux combination $f_{1}{ }^{12}=3 m$ and $f^{2}=-m$. The non-vanishing $f^{2}$ should again be compensated by a non-trivial dilaton anzats. For the case at hand, for which only $\left(U^{-1}\right)_{i a}=0$ ( and still $\left.\partial_{i}\left(U^{-1}\right)^{i}{ }_{a}=0\right)$, the equation (3.27) corresponding to $\eta^{A}=0$ gives the following set of equations:

$$
\begin{aligned}
\left(U^{-1}\right)^{i}{ }_{a} \partial_{i} \lambda & =0 \\
\partial_{1}\left(U^{-1}\right)^{1 a}+\tilde{\partial}^{1}\left(U^{-1}\right)_{1}{ }^{a}-2\left(U^{-1}\right)^{i a} \partial_{i} \lambda-2\left(U^{-1}\right)_{i}{ }^{a} \tilde{\partial}^{i} \lambda & =0 .
\end{aligned}
$$

It is easily checked that

$$
\lambda=\frac{m \tilde{x}_{2}}{2}
$$

solves these equations. Therefore, the anzats for the generalized dilaton is

$$
d(X, Y)=d(X)+\frac{m \tilde{x}_{2}}{2} .
$$

Although this configuration generates the same type of gauging, the total number of coordinates that the fields on the total space can depend on are different. This time, the number of allowed coordinates is 11: $(Y, X)=\left(\left\{\tilde{x}_{1}, \tilde{x}_{2}, x^{1}\right\},\left\{x^{3}, \ldots, x^{10}\right\}\right)$. The first 3 coordinates $\left\{\tilde{x}_{1}, \tilde{x}_{2}, x^{1}\right\}$ are the non-dynamical internal $Y$ coordinates and the remaining 8 coordinates are the dynamical external $X$ coordinates.

We would like to remark that this type of degeneracy in the duality twisted reductions of DFT was also noted in [50]. Indeed, it was observed in [50] that two inequivalent twists may generate the same gauging. Moreover, they also showed that it was possible for one of these twists to be geometrical, in the sense that it respects the strong and the weak constraints and yet the other might be non-geometrical, violating both of the constraints in the internal doubled space. The phenomena we observe here is exactly the same. Although they generate the same gauging, the twist in section 3.2.1 and the mixed twists we have considered here have a remarkable difference. The twist in 3.2.1 respects both the weak and the strong constraints on the total space. However, both constraints are violated in the doubled internal space for the mixed twists we have considered here. Indeed,

$$
\partial^{P} \partial_{P} U_{M}^{A}=0
$$


is not satisfied by one of the components of the twist matrix $U$ in both cases. This is most easily seen, when one writes down the twist matrix explicitly, as we do in appendix B (for the first example of this section with trivial dilaton anzats). The examples we have studied here is a nice demonstration of the fact that the weak constraint is stronger than the consistency conditions of duality twisted reductions of DFT, as was discussed in [27].

\subsection{The reduced action}

In the previous section, we have identified the twists which give rise to a deformation of the form

$$
\not \partial \chi(X, Y) \rightarrow S\left(\tilde{\not} \chi(X)+F_{0}\right),
$$

where the 0 -form $F_{0}$ comes from contractions of $\alpha$ and $\chi .{ }^{13}$ This type deformation can occur only in Type IIA, where $\chi$ has negative chirality (and hence corresponds to a polyform consisting of odd differential forms), as such deformations arose from contractions of a 1form or a 3-form, which only exists in Type IIA. Also note that $F_{0}$ need not be a constant form, as it might include a part coming from the contractions of the spinor field $\chi$. In what follows, we will assume that this is not the case (which is trivially satisfied if, for example, $\chi(X, Y)$ has no components along the direction in $x^{2}$, as discussed in the previous sections). Hence, $F_{0}$ is a constant form. Furthermore, this assumption implies that $\tilde{\not} \chi=\not \nabla \chi=\not \partial \chi$.

Let us plug in (3.41) and (2.17) into the DFT Lagrangian for the RR sector (3.6).

$$
\begin{aligned}
L & =\frac{1}{4}\left\langle S(Y)\left(\not \partial \chi(X)+F_{0}\right), C^{-1}\left(S^{-1}\right)^{\dagger}(Y) \mathbb{S} S^{-1}(Y) S(Y)\left(\not \partial \chi(X)+F_{0}\right)\right\rangle \\
& =\frac{1}{4}\left\langle S(Y)\left(\not \partial \chi(X)+F_{0}\right), C^{-1}\left(S^{-1}\right)^{\dagger}(Y) S_{b}^{\dagger} S_{g}^{-1} S_{b}\left(\not \partial \chi(X)+F_{0}\right)\right\rangle \\
& =\frac{1}{4}\left\langle S(Y)\left(\not \partial \chi(X)+F_{0}\right), S(Y) S_{b}^{-1} C^{-1} S_{g}^{-1} S_{b}\left(\not \partial \chi(X)+F_{0}\right)\right\rangle \\
& =\frac{1}{4}\left\langle S_{b}\left(\not \partial \chi(X)+F_{0}\right), C^{-1} S_{g}^{-1} S_{b}\left(\not \partial \chi(X)+F_{0}\right)\right\rangle \\
& =\frac{1}{4}\left(F+e^{-B} \wedge F_{0}\right) \wedge *\left(F+e^{-B} \wedge F_{0}\right),
\end{aligned}
$$

where $F=S_{b}(\not \partial \chi(X))$. This is the Lagrangian of the massive IIA theory [21, 43], as was discussed in section 3. Note that in the second line above we used the definition $\mathbb{S}=S_{b}^{\dagger} S_{g}^{-1} S_{b}$, whereas in the third line we used $C^{-1}\left(S^{-1}\right)^{\dagger}(Y)=S(Y) C^{-1}$ and $S_{b}^{-1} C^{-1}=$ $C^{-1} S_{b}^{\dagger}$ (since both $S(Y)$ and $S_{b}$ are elements of $\operatorname{Spin}^{+}(d, d)$ ) and finally in the fourth line we used the invariance property of the Mukai pairing under $\operatorname{Spin}^{+}(d, d)$ transformations.

The reduced self-duality condition (2.36) gives

$$
\not \partial \chi(X)+F_{0}=-C^{-1} \mathbb{S}\left(\not \partial \chi(X)+F_{0}\right)
$$

which, in terms of the p-form components $F_{p}^{m}$ of the spinor field $\not \partial \chi+F_{0}$, is equivalent to

$$
F_{8-p}^{m}=(-1)^{\left[\frac{p-1}{2}\right]} * F_{p}^{m} .
$$

\footnotetext{
${ }^{13}$ We define $\tilde{\not}$ such that $\tilde{\not} \chi(X)=\not \nabla \chi(X)-G_{0}$, where $G_{0}$ is the 0 -form field that comes from the contractions of $\chi$.
} 
The field equations arising from (3.42) must be supplemented by (3.44), where $*$ is the Hodge opearator with respect to the metric $g(X)$. Note that the top degree form in (3.44) is an 8-form, as we assume that the components of all forms along the directions $x^{1}, x^{2}$ have been integrated out. Also note that, even for the case of non-constant $F_{0}$, which could be generated by non-zero contractions of $\chi$, the field equation $d * F_{8}=0,{ }^{14}$ would have forced its dual $F_{0}$ to be constant, as discussed in section 3 .

\section{Conclusion and outlook}

In this paper, we considered the possibility of obtaining massive deformations of Type IIA theory through a duality twisted reduction in the RR sector. This is motivated by a paper of Hohm and Kwak [21], where they obtain massive IIA within DFT, via a linear dependence of the spinor field $\chi$ (which encodes the p-form fields) on one of the dual coordinates. Here, we allow all the fields in the theory to depend on (some of) the dual coordinates through a duality twisted anzats. We show that a 0-form field strength (and hence a mass parameter) can be generated for certain choices of the twist element $S(\tilde{y}, y)$. The twist elements should be chosen such that they deform the differential form, which encodes the field strengths of the (modified) RR gauge potentials by a 0 -form. The gauge potential of such a 0 -form field could only be a (-1)-form. This is in line with the interpretation of [21] that a (-1)-form is a 1-form depending on the dual coordinates.

One interesting aspect of our anzats is that for some choices of the twist element $S(\tilde{y}, y)$, both the strong and the weak constraints are violated explicitly in the internal doubled space. Let us call such twists, following [50], non-geometric twists. The natural question that arises is whether the deformations/gaugings we obtain in lower dimensions through such non-geometric twists can be obtained from T-duals of a conventional compactification of supergravity. In order to discuss this issue, it is useful to introduce some more terminology from [50], where gaugings obtained from duality twisted reductions of DFT was classified. Two important notions from [50] are the twist orbit and the orbit of gaugings. Twist orbit is defined to be the set of twist matrices connected by T-duality transformations and likewise, the orbit of gaugings is defined as the set of gaugings that are related by duality transformations. The importance of these definitions lie in the fact that two twists that lie in the same twist orbit generate gaugings belonging to the same orbit of gaugings and two theories that belong to the same orbit of gaugings are physically equivalent. ${ }^{15}$ It is then natural to call a orbit of gauging geometric, if it includes at least one representative gauging that can be obtained through a conventional compactification. Now the question we posed above can be paraphrased using this terminology: "Do the theories that we have obtained here belong to a geometric or a non-geometric orbit?"

\footnotetext{
${ }^{14}$ Note that the field equation of the 7 -form potential must be $d * F_{8}=0$, as the only possible coupling of it can be with the B-field, which would give a 9-form that vanishes in 8 dimensions. This is also what happens in 10 dimension, where the field equation of the 9 -form potential gives $d * F_{10}=0$, whereas the other field equations will be of the form $d * F_{2 n}+d B \wedge * F_{2 n+2}=0$.

${ }^{15}$ Note that the reverse argument of the first part of this sentence is not necessarily true. Two twists that lie in different twist orbits may generate gaugings in the same orbit of gauging, as we will discuss below. This possibility was also emphasized in [50].
} 
The condition (2.29) was identified in [50] as a criterion to label the orbits of gaugings as geometric or non-geometric and it was shown that gaugings that do not satisfy (2.29) are non-geometric and require a truly doubled background in order to be lifted to a compactification of DFT. On the other hand, a geometric twist, which automatically satisfies (2.29), would always give rise to a theory that lies in a geometric orbit of gauging [50]. Indeed, if the strong and the weak constraints are not violated, it is always possible to T-dualize to a frame in which the fields and the gauge parameters of the theory have no dependence on the dual coordinates [4]. Accordingly, the twists we considered in sections 3.2.1 and 3.2.2, being geometric twists (as they do not violate the strong and weak constraints of DFT even in the internal space), cannot give rise to a theory that lies in a non-geometric orbit of gauging, despite the appearance of the Q- and R-fluxes. What about the twists we considered in section 3.2.3? Such twists belong to an interesting class, as they violate the strong and the weak constraints explicitly, and hence they are non-geometric, and yet they satisfy the constraint (2.29). For the example we encounter in section 3.2.3, we immediately see that, the resulting theory belongs to a geometric orbit of gauging, as the deformation it gives rise to is exactly the same as the deformation in section 3.2.1. This is a phenomenon, which has already been discussed and exemplified in [50]. Indeed, it was observed in [50] too that twists that belong to different twist orbits may generate the same gauging, even when one of these orbits is geometric and the other is non-geometric, violating both of the constraints in the internal doubled space. The phenomena we observe here is exactly the same. Therefore, we conclude that all the solutions we obtain here, which are massive deformations of Type II supergravity theories with various type of fluxes belong to a geometric orbit of gauging and hence can be T-dualized to conventional compactifications of Type II massive supergravity.

Another interesting aspect of our work is that, for some choices of the twist $S(\tilde{y}, y)$, requirement of consistency forces the dilaton field to pick up a linear dependence one of the dual coordinates, through an anzats of the form $\phi(X, Y)=\phi(X)+\rho(\tilde{y})$. This makes us wonder about the possible connections between the field equations following from such reductions and the recently discovered generalized supergravity equations [37]. The reason one might hope for such a connection is the recent works [38-40], where it was shown that generalized supergravity equations can be obtained from DFT (in [40]) and from exceptional field theory (EFT) ${ }^{16}$ (in [38]) through an anzats by which the dilaton acquires a linear dependence on the dual coordinates. On the other hand, it is interesting to note that the dilaton $\phi$ in the ordinary Type IIA supergravity action (in the string frame) has a shift symmetry, which also allows the following Scherk-Schwarz type anzats for $\phi: \phi(x, y)=$ $\phi(x)+\rho(y)$, where $y$ are the coordinates of the internal manifold. In the papers $[51,52]$, such a reduction to 4 dimensions with liner dependence on $y$ was considered. The resulting theory is a massive, gauged theory and its Lagrangian can be put in the general form given by Schön and Weidner in [53], where (part of) the $\operatorname{SL}(2)$ of the global $\mathrm{SL}(2) \times \operatorname{SO}(6,6)$ group was gauged. It would be interesting to explore the type of gaugings that would arise in four dimensions through a duality twisted reduction with twists involving $\rho=\rho(y, \tilde{y})$.

\footnotetext{
${ }^{16}$ Exceptional Field Theory is a U-duality covariant extension of supergravity.
} 
We would like to mention that massive deformations of Type IIA theory has also been studied by various groups within the context of EFT [54, 55]. In [55], massive Type IIA theory arises as a purely geometric solution of a consistent deformation of EFT, which is called XFT (referring to X-deformed EFT). The deformation of EFT is based on a modification of the generalised Lie derivative by non-derivative terms of the form

$$
\tilde{\mathcal{L}}_{\Lambda}=\mathcal{L}_{\Lambda}+\Lambda^{M} X_{M}
$$

where $X_{M}$ take values in the Lie algebra of the U-duality group. Acting on a field in a representation of the U-duality group, it takes the form $\left(X_{M}\right)_{N}^{P}=X_{M N}^{P}$. The deformation is consistent only if $X_{M N}^{P}$ satisfy a set of constraints. Namely, one should have

$$
X_{M P}^{R} X_{N R}^{Q}-X_{N P}^{R} X_{M R}^{Q}+X_{M N}^{R} X_{R P}^{Q} \quad \text { and } \quad X_{M N}^{P} \partial_{P}=0 .
$$

It is interesting to note that the duality twisted anzats we have studied in this paper also induce non-derivative deformations of a similar type on the Lie derivative and the Dirac operator, and the fluxes $f_{M N}^{P}$ which determine this deformation should obey exactly the same type of constraints, as listed in (2.24) and (2.25).

\section{Acknowledgments}

This work is supported by the Turkish Council of Research and Technology (TÜBITTAK) through the ARDEB 1001 project with grant number 114F321, in conjunction with the COST action MP1405 QSPACE.

\section{A The NS-NS sector}

In this appendix, we review briefly the duality twisted reductions of the DFT of the NS-NS sector of string theory. Our review follows closely [27]. Let us begin by presenting the generalized Ricci scalar $\mathcal{R}(\mathcal{H}, d)$, that determines the action (2.4).

$$
\begin{aligned}
\mathcal{R}(\mathcal{H}, d)= & 4 \mathcal{H}^{M N} \partial_{M} \partial_{N} d-\partial_{M} \partial_{N} \mathcal{H}^{M N}-4 \mathcal{H}^{M N} \partial_{M} d \partial_{N} d+4 \partial_{M} \mathcal{H}^{M N} \partial_{N} d \\
& +\frac{1}{8} \mathcal{H}^{M N} \partial_{M} \mathcal{H}^{K L} \partial_{N} \mathcal{H}_{K L}-\frac{1}{2} \mathcal{H}^{M N} \partial_{M} \mathcal{H}^{K L} \partial_{K} \mathcal{H}_{N L} \\
& +\frac{1}{2} \partial_{M} \varepsilon^{a}{ }_{P} \partial^{M} \varepsilon^{b}{ }_{Q} S_{a b} \eta^{P Q}
\end{aligned}
$$

Here $\mathcal{H}$ is the generalized metric and $\varepsilon^{a}{ }_{P}$ is the generalized vielbein with $\mathcal{H}_{M N}=\varepsilon^{a}{ }_{M} S_{a b} \varepsilon^{b}{ }_{N}$, where $S_{a b}=\operatorname{diag}(-1,1, \cdots, 1 ;-1,1, \cdots, 1)$ is the planar metric. The term in the last line is not in the original generalized metric formulation of DFT and vanishes when the strong constraint is imposed [27]. When the strong constraint is satisfied, the action (2.3) is invariant under the following gauge transformations, which forms a gauge algebra that is closed with respect to the C-bracket.

$$
\begin{aligned}
\delta_{\xi} \mathcal{H}_{M N} & =\widehat{\mathcal{L}}_{\xi} \mathcal{H}_{M N} \\
& \equiv \xi^{P} \partial_{P} \mathcal{H}_{M N}+\left(\partial_{M} \xi^{P}-\partial^{P} \xi_{M}\right) \mathcal{H}_{P N}+\left(\partial_{N} \xi^{P}-\partial^{P} \xi_{N}\right) \mathcal{H}_{M P}, \\
\delta d & =\xi^{M} \partial_{M} d-\frac{1}{2} \partial_{M} \xi^{M}
\end{aligned}
$$


After applying the reduction anzats (2.20), (2.19) the generalized Ricci scalar is deformed as $\mathcal{R} \rightarrow \mathcal{R}_{\text {def }}$ with

$$
\begin{aligned}
\mathcal{R}_{\mathrm{def}}= & \mathcal{R}+\mathcal{R}_{f}, \\
\mathcal{R}_{f}= & -\frac{1}{2} f_{B C}^{A} \mathcal{H}_{B D} \mathcal{H}^{C E} \partial_{D} \mathcal{H}_{A E}-\frac{1}{12} f_{B C}^{A} f_{E F}^{D} \mathcal{H}_{A D} \mathcal{H}^{B E} \mathcal{H}^{C F} \\
& -\frac{1}{4} f_{B C}^{A} f_{A D}^{B} \mathcal{H}^{C D}-2 \eta_{A} \partial_{B} \mathcal{H}^{A B}+4 \eta_{A} \mathcal{H}^{A B} \partial_{B} d-\eta_{A} \eta_{B} \mathcal{H}^{A B},
\end{aligned}
$$

which determines an action closed under the following deformed gauge transformation rules

$$
\begin{aligned}
\hat{\delta}_{\hat{\xi}} \mathcal{H}_{A B} & =\delta_{\hat{\xi}} \mathcal{H}_{A B}-f_{A C D} \hat{\xi}^{C} \mathcal{H}_{B}^{D}+f_{C B}^{D} \hat{\xi}^{C} \mathcal{H}_{A D} \\
\hat{\delta}_{\hat{\xi}} & =\delta_{\hat{\xi}} d-\frac{1}{2} \eta_{A} \hat{\xi}^{A} .
\end{aligned}
$$

Here $\xi^{M}(X, Y)=\left(U^{-1}\right)_{A}^{M} \hat{\xi}^{A}(X)$, as in section 2. As emphasized in section 2, the closure of gauge algebra now requires the imposition of the strong and weak constraints only in the external space. In the following subsections, we will analyze the deformations induced in the NS-NS sector by the twists in subsections 3.2.1 and 3.2.2.

\section{A.1 Twists with non-vanishing $f_{1}^{12}$}

Consider the twists in sections 3.2.1 and 3.2.3. They all give rise to the same deformation with the only non-vanishing flux component $f_{1}^{12}$, with all other flux components zero (except the ones, obviously, related to $f_{1}^{12}$ by permutations of the indices). Plugging $f_{1}^{12}=m$ in (A.3) one finds

$$
\mathcal{R}_{f}=-\frac{1}{2} m \mathcal{H}_{[1}{ }^{d} \mathcal{H}_{2] E} \partial_{d} \mathcal{H}_{1}{ }^{E}-\frac{1}{6} m^{2} \mathcal{H}^{11}\left(\mathcal{H}_{11} \mathcal{H}_{22}-\left(\mathcal{H}_{12}\right)^{2}\right)-\frac{1}{2} m^{2} \mathcal{H}_{22}
$$

One can write this in terms of the metric and B-field elements by using the following parametrization of the generalized metric

$$
\begin{array}{rlrl}
\mathcal{H}_{a b} & =g_{a b}-B_{a c} g^{c d} B_{d b} & & \\
\mathcal{H}_{a}^{b} & =B_{a c} g^{c b}, & \mathcal{H}^{a}{ }_{b}=-g^{a c} B_{c b} \\
\mathcal{H}^{a b} & =g^{a b} . &
\end{array}
$$

Recall that the fields in the resulting theory cannot have dependence on the coordinates $x^{1}, x^{2}$, so $\partial_{d}$ is non-zero only if $d \neq 1,2$.

One can also compute the deformed gauge transformation rules and finds the follow$\operatorname{ing}^{17}$

$$
\begin{aligned}
\delta g_{11} & =\mathcal{L}_{\xi} g_{11}+2 m\left(g_{11} B_{12} \xi^{1}-g_{12} \tilde{\xi}_{1}+g_{11} \tilde{\xi}_{2}\right) \\
\delta g_{12} & =\mathcal{L}_{\xi} g_{12}+2 m g_{12} B_{12} \xi^{1}+m g_{12} \tilde{\xi}_{2}-m g_{22} \tilde{\xi}_{1}, \\
\delta g_{22} & =\mathcal{L}_{\xi} g_{22}+2 m g_{22} B_{12} \xi^{1} \\
\delta g_{a b} & =\mathcal{L}_{\xi} g_{a b}+m\left(g_{1(a} B_{b) 2}-g_{2(a} B_{b) 1}\right) \xi^{1}, \quad a, b \neq 1,2 \\
\delta B_{12} & =\mathcal{L}_{\xi} B_{12}+\partial_{[1} \tilde{\xi}_{2]}+m B_{12} \tilde{\xi}_{2}-m\left(g_{11} g_{22}-\left(g_{12}\right)^{2}-\left(B_{12}\right)^{2}\right) \xi^{1} \\
\delta B_{a b} & =\mathcal{L}_{\xi} B_{a b}+\partial_{[a} \tilde{\xi}_{b]}-m\left(g_{a 1} g_{2 b}-g_{a 2} g_{1 b}+B_{a 1} B_{2 b}-B_{a 2} B_{1 b}\right) \xi^{1}, \quad a, b \neq 1,2
\end{aligned}
$$

where $\mathcal{L}_{\xi} u_{i j} \equiv \xi^{p} \partial_{p} u_{i j}+\partial_{i} \xi^{p} u_{p j}+\partial_{j} \xi^{p} u_{i p}$.

\footnotetext{
${ }^{17}$ Note that we have omitted the hats on the gauge parameters for simplicity, so that $\hat{\xi}^{A}=\left(\tilde{\xi}_{i}, \xi^{i}\right)$.
} 


\section{A.2 Twists with non-vanishing $f^{123}$}

Consider the twist in section 3.2.2. Recall that the fluxes induced by this twist are $f^{123}=m$. Then the deformation in the generalized Ricci scalar is

$$
\mathcal{R}_{f}=-\frac{1}{2} m \mathcal{H}_{[1}{ }^{d} \mathcal{H}_{2}{ }^{E} \partial_{d} \mathcal{H}_{3] E}-\frac{1}{12} m^{2} \operatorname{det} \mathcal{H}_{i j},
$$

where $\operatorname{det} \mathcal{H}_{i j}$ is the determinant of the $3 \times 3$ matrix whose components are $\mathcal{H}_{i j}$ with $i, j=1,2,3$. Note that the direction $x^{d}$ appearing in $\partial_{d}$ in the first term cannot include the directions $x^{i}, i=1,2,3$, as consistency requires that the fields of the reduced theory should not depend on these coordinates. The deformed gauge transformation rules are

$$
\begin{aligned}
\delta g_{i j} & =-m g_{i[1} B_{2 j} \tilde{\xi}_{3]}-m g_{j[1} B_{2 \underline{i}} \tilde{\xi}_{3]} \\
\delta B_{i j} & =-m\left(g_{i[1} g_{2 \underline{k}}+B_{i[1} B_{2 \underline{k}}\right) \tilde{\xi}_{3]}
\end{aligned}
$$

Here, the underlined indices are not to be antisymmetrized.

\section{B The mixed twist matrix}

We present here the explicit form of the twist matrix of section 3.2.3 (the first one with trivial dilaton anzats).

$$
\begin{aligned}
& \left(U^{-1}\right)_{A}^{M}=\left(\begin{array}{cc}
\left(U^{-1}\right)_{i}{ }^{a} & \left(U^{-1}\right)_{i a} \\
\left(U^{-1}\right)^{i a} & \left(U^{-1}\right)^{i}{ }_{a}
\end{array}\right)
\end{aligned}
$$

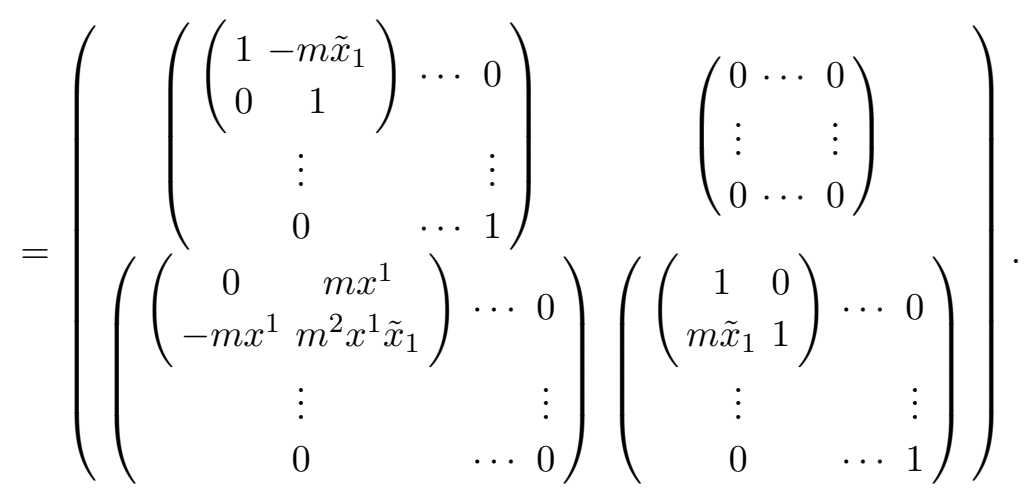

Open Access. This article is distributed under the terms of the Creative Commons Attribution License (CC-BY 4.0), which permits any use, distribution and reproduction in any medium, provided the original author(s) and source are credited.

\section{References}

[1] C. Hull and B. Zwiebach, Double Field Theory, JHEP 09 (2009) 099 [arXiv:0904.4664] [INSPIRE].

[2] C. Hull and B. Zwiebach, The gauge algebra of double field theory and Courant brackets, JHEP 09 (2009) 090 [arXiv: 0908.1792] [InSPIRE]. 
[3] O. Hohm, C. Hull and B. Zwiebach, Background independent action for double field theory, JHEP 07 (2010) 016 [arXiv: 1003.5027] [INSPIRE].

[4] O. Hohm, C. Hull and B. Zwiebach, Generalized metric formulation of double field theory, JHEP 08 (2010) 008 [arXiv: 1006.4823] [INSPIRE].

[5] A.A. Tseytlin, Duality symmetric closed string theory and interacting chiral scalars, Nucl. Phys. B 350 (1991) 395 [INSPIRE].

[6] A.A. Tseytlin, Duality Symmetric Formulation of String World Sheet Dynamics, Phys. Lett. B 242 (1990) 163 [INSPIRE].

[7] W. Siegel, Two vierbein formalism for string inspired axionic gravity, Phys. Rev. D 47 (1993) 5453 [hep-th/9302036] [INSPIRE].

[8] W. Siegel, Superspace duality in low-energy superstrings, Phys. Rev. D 48 (1993) 2826 [hep-th/9305073] [INSPIRE].

[9] W. Siegel, Manifest duality in low-energy superstrings, hep-th/9308133 [INSPIRE].

[10] C.M. Hull, A geometry for non-geometric string backgrounds, JHEP 10 (2005) 065 [hep-th/0406102] [INSPIRE].

[11] A. Dabholkar and C. Hull, Generalised T-duality and non-geometric backgrounds, JHEP 05 (2006) 009 [hep-th/0512005] [INSPIRE].

[12] C.M. Hull, Doubled Geometry and T-Folds, JHEP 07 (2007) 080 [hep-th/0605149] [INSPIRE].

[13] C.M. Hull and R.A. Reid-Edwards, Gauge symmetry, T-duality and doubled geometry, JHEP 08 (2008) 043 [arXiv: 0711.4818] [INSPIRE].

[14] N. Hitchin, Lectures on generalized geometry, arXiv:1008.0973 [INSPIRE].

[15] N. Hitchin, Generalized Calabi-Yau manifolds, Quart. J. Math. 54 (2003) 281 [math/0209099] [INSPIRE].

[16] M. Gualtieri, Generalized complex geometry, math/0401221 [INSPIRE].

[17] O. Hohm, S.K. Kwak and B. Zwiebach, Double Field Theory of Type II Strings, JHEP 09 (2011) 013 [arXiv:1107.0008] [INSPIRE].

[18] O. Hohm, S.K. Kwak and B. Zwiebach, Unification of Type II Strings and T-duality, Phys. Rev. Lett. 107 (2011) 171603 [arXiv:1106.5452] [INSPIRE].

[19] I. Jeon, K. Lee, J.-H. Park and Y. Suh, Stringy Unification of Type IIA and IIB Supergravities under $N=2 D=10$ Supersymmetric Double Field Theory, Phys. Lett. B 723 (2013) 245 [arXiv:1210.5078] [inSPIRE].

[20] I. Jeon, K. Lee and J.-H. Park, Ramond-Ramond Cohomology and $\mathrm{O}(D, D)$ T-duality, JHEP 09 (2012) 079 [arXiv: 1206.3478] [INSPIRE].

[21] O. Hohm and S.K. Kwak, Massive Type II in Double Field Theory, JHEP 11 (2011) 086 [arXiv: 1108.4937] [INSPIRE].

[22] L.J. Romans, Massive N=2a Supergravity in Ten-Dimensions, Phys. Lett. B 169 (1986) 374 [INSPIRE].

[23] J. Scherk and J.H. Schwarz, How to Get Masses from Extra Dimensions, Nucl. Phys. B 153 (1979) 61 [INSPIRE]. 
[24] J. Scherk and J.H. Schwarz, Spontaneous Breaking of Supersymmetry Through Dimensional Reduction, Phys. Lett. B 82 (1979) 60 [InSPIRE].

[25] D. Geissbuhler, Double Field Theory and $N=4$ Gauged Supergravity, JHEP 11 (2011) 116 [arXiv: 1109.4280] [INSPIRE].

[26] G. Aldazabal, W. Baron, D. Marques and C. Núñez, The effective action of Double Field Theory, JHEP 11 (2011) 052 [Erratum ibid. 11 (2011) 109] [arXiv:1109.0290] [INSPIRE].

[27] M. Graña and D. Marques, Gauged Double Field Theory, JHEP 04 (2012) 020 [arXiv:1201.2924] [INSPIRE].

[28] D. Geissbuhler, D. Marques, C. Núñez and V. Penas, Exploring Double Field Theory, JHEP 06 (2013) 101 [arXiv: 1304.1472] [INSPIRE].

[29] W. Cho, J.J. Fernández-Melgarejo, I. Jeon and J.-H. Park, Supersymmetric gauged double field theory: systematic derivation by virtue of twist, JHEP 08 (2015) 084 [arXiv: 1505.01301] [INSPIRE].

[30] D.S. Berman and K. Lee, Supersymmetry for Gauged Double Field Theory and Generalised Scherk-Schwarz Reductions, Nucl. Phys. B 881 (2014) 369 [arXiv:1305.2747] [INSPIRE].

[31] F. Hassler and D. Lüst, Consistent Compactification of Double Field Theory on Non-geometric Flux Backgrounds, JHEP 05 (2014) 085 [arXiv:1401.5068] [INSPIRE].

[32] R. Blumenhagen, F. Hassler and D. Lüst, Double Field Theory on Group Manifolds, JHEP 02 (2015) 001 [arXiv: 1410.6374] [INSPIRE].

[33] R. Blumenhagen, P. du Bosque, F. Hassler and D. Lüst, Generalized Metric Formulation of Double Field Theory on Group Manifolds, JHEP 08 (2015) 056 [arXiv:1502.02428] [INSPIRE].

[34] A. Çatal-Özer, Duality Twisted Reductions of Double Field Theory of Type II Strings, JHEP 09 (2017) 044 [arXiv: 1705.08181] [INSPIRE].

[35] G. Aldazabal, D. Marques, C. Núñez and J.A. Rosabal, On type IIB moduli stabilization and $N=4$, 8 supergravities, Nucl. Phys. B 849 (2011) 80 [arXiv:1101.5954] [INSPIRE].

[36] G. Dibitetto, A. Guarino and D. Roest, How to halve maximal supergravity, JHEP 06 (2011) 030 [arXiv: 1104.3587] [INSPIRE].

[37] G. Arutyunov, S. Frolov, B. Hoare, R. Roiban and A.A. Tseytlin, Scale invariance of the $\eta$-deformed $A d S_{5} \times S^{5}$ superstring, T-duality and modified type-II equations, Nucl. Phys. B 903 (2016) 262 [arXiv:1511.05795] [INSPIRE].

[38] A. Baguet, M. Magro and H. Samtleben, Generalized IIB supergravity from exceptional field theory, JHEP 03 (2017) 100 [arXiv: 1612.07210] [INSPIRE].

[39] Y. Sakatani, S. Uehara and K. Yoshida, Generalized gravity from modified DFT, JHEP 04 (2017) 123 [arXiv:1611.05856] [INSPIRE].

[40] J.-i. Sakamoto, Y. Sakatani and K. Yoshida, Weyl invariance for generalized supergravity backgrounds from the doubled formalism, PTEP 2017 (2017) 053B07 [arXiv:1703.09213] [INSPIRE].

[41] S. Mukai, Symplectic Structure of the Moduli Space of Sheaves on an Abelian or K3 Surface, Invent. Math. 77 (1984) 101.

[42] M. Fukuma, T. Oota and H. Tanaka, Comments on T dualities of Ramond-Ramond potentials on tori, Prog. Theor. Phys. 103 (2000) 425 [hep-th/9907132] [INSPIRE]. 
[43] E. Bergshoeff, R. Kallosh, T. Ortín, D. Roest and A. Van Proeyen, New formulations of $D=10$ supersymmetry and D8-O8 domain walls, Class. Quant. Grav. 18 (2001) 3359 [hep-th/0103233] [INSPIRE].

[44] J. Polchinski, Dirichlet Branes and Ramond-Ramond charges, Phys. Rev. Lett. 75 (1995) 4724 [hep-th/9510017] [INSPIRE].

[45] I.V. Lavrinenko, H. Lü, C.N. Pope and K.S. Stelle, Superdualities, brane tensions and massive IIA/IIB duality, Nucl. Phys. B 555 (1999) 201 [hep-th/9903057] [INSPIRE].

[46] D. Andriot, O. Hohm, M. Larfors, D. Lüst and P. Patalong, A geometric action for non-geometric fluxes, Phys. Rev. Lett. 108 (2012) 261602 [arXiv:1202.3060] [INSPIRE].

[47] D. Andriot, O. Hohm, M. Larfors, D. Lüst and P. Patalong, Non-Geometric Fluxes in Supergravity and Double Field Theory, Fortsch. Phys. 60 (2012) 1150 [arXiv:1204.1979] [INSPIRE].

[48] J. Shelton, W. Taylor and B. Wecht, Nongeometric flux compactifications, JHEP 10 (2005) 085 [hep-th/0508133] [INSPIRE].

[49] C.M. Hull, Massive string theories from M-theory and F-theory, JHEP 11 (1998) 027 [hep-th/9811021] [INSPIRE].

[50] G. Dibitetto, J.J. Fernández-Melgarejo, D. Marques and D. Roest, Duality orbits of non-geometric fluxes, Fortsch. Phys. 60 (2012) 1123 [arXiv:1203.6562] [INSPIRE].

[51] J.-P. Derendinger, P.M. Petropoulos and N. Prezas, Axionic symmetry gaugings in $N=4$ supergravities and their higher-dimensional origin, Nucl. Phys. B 785 (2007) 115 [arXiv: 0705.0008] [INSPIRE].

[52] A. Çatal-Özer, C. Deliduman and E. Ulas Saka, A massive S-duality in 4 dimensions, JHEP 12 (2011) 102 [arXiv:1110.4974] [INSPIRE].

[53] J. Schön and M. Weidner, Gauged $N=4$ supergravities, JHEP 05 (2006) 034 [hep-th/0602024] [INSPIRE].

[54] F. Ciceri, A. Guarino and G. Inverso, The exceptional story of massive IIA supergravity, JHEP 08 (2016) 154 [arXiv: 1604.08602] [INSPIRE].

[55] D. Cassani, O. de Felice, M. Petrini, C. Strickland-Constable and D. Waldram, Exceptional generalised geometry for massive IIA and consistent reductions, JHEP 08 (2016) 074 [arXiv: 1605.00563] [INSPIRE]. 